\title{
Border collision bifurcations of stroboscopic maps in periodically driven spiking models*
}

\author{
A. Granados M. Krupa F. Clément
}

\begin{abstract}
In this work we consider a general non-autonomous hybrid system based on the integrate-and-fire model, widely used as simplified version of neuronal models and other types of excitable systems. Our unique assumption is that the system is monotonic, possesses an attracting subthreshold equilibrium point and is forced by means of periodic pulsatile (square wave) function.

In contrast to classical methods, in our approach we use the stroboscopic map (time- $T$ return map) instead of the so-called firing-map. It becomes a discontinuous map potentially defined in an infinite number of partitions. By applying theory for piecewise-smooth systems, we avoid relying on particular computations and we develop a novel approach that can be easily extended to systems with other topologies (expansive dynamics) and higher dimensions.

More precisely, we rigorously study the bifurcation structure in the twodimensional parameter space formed by the amplitude and the duty cycle of the pulse. We show that it is covered by regions of existence of periodic orbits given by period adding structures. They do not only completely describe all the possible spiking asymptotic dynamics but also the behavior of the firing rate, which is a devil's staircase as a function of the parameters.
\end{abstract}

\section{Introduction}

In the context of neuronal modeling (and similarly for hormone release), one relies on systems that are able to exhibit large-amplitude responses under certain stimuli. An example of such behavior is an action potential (AP) or spike of a neuron. There exist accurate but quite complex models of APs, e.g. the Hudgkin-Huxley equations HH52. Simpler models are the Morris-Lecar ML81] or the FitzHugh and Nagumo Fit61, NAY62. All these models use the principle of excitability, and often exhibit slow/fast dynamics.

Hybrid systems with resets are used as approximate models of excitability, with

*This work has been financially supported by the Large Scale Initiative Action REGATE, by the Spanish MINECO-FEDER Grants MTM2009-06973, MTM2012-31714 and the Catalan Grant 2009SGR859 
the discontinuity, that is the reset, used to mimic the spike. In many contexts a spike occurs when the system receives a sufficient amount of stimulus.

One can distinguish between different types of systems with resets. The simplest are integrate-and-fire models, for which the differential equation exhibits very simple dynamics (it is constant or linear). Other models include more complicated subthreshold dynamics including instabilities (which introduce expanding dynamics) in order to provide more realistic representations of the underlying phenomena. This is the case of the so-called Izhikevich model [Izh03. Other approaches BG05, TB09, MHR12] let the threshold depend on the state variable (thus increasing the dimension of the system) in order to obtain a more accurate model with possibly more complex properties, as is the case of type III neurons CPF08, Izh07.

Due to the discontinuity in the trajectories given by the reset, one cannot directly apply classical theory for smooth systems in order to study these type of excitable systems. A typical approach to overcome this problem is to consider the so-called adaption, firing or impact map, which is a Poincaré map onto the threshold [KHR81, CB99, CTW12, TB09, JMB ${ }^{+} 13$, DKTG12]; that is, one takes an initial condition on the threshold (which leads to a reset) and one then integrates the system until the threshold is reached again. This is a well known technique also in other disciplines involving piecewise-smooth dynamics (see dBBCK08 for examples); this map becomes the composition of smooth maps and hence provides a regular version of the system which can be studied by means of classical tools for smooth systems.

Note that, in order to compute the firing map, one needs to know the time needed by the system to perform the next spike (firing times). Even assuming linearity, these times need to be computed numerically, as they involve transcendental equations. Hence, one also relies on numerical computations in order to derive properties of the firing map.

When introducing a $T$-periodic forcing to the system (a periodic current in the neuronal context), the firing map must include time as a variable. In fact, for the one-dimensional case, it becomes a map that returns the next firing time. Hence, in order to study the existence of periodic orbits, explicit knowledge of the behaviour of these times becomes crucial, as one needs to check whether the difference between consecutive spiking times is congruent (rational multiple) with $T$. Results in the context of firing maps have been obtained in specific cases. For example, it was shown in KHR81 that, for a linear system, by using the explicit expression for the flow, one can approximate the firing map by a certain a circle map and derive information about rotation and firing numbers of existing periodic orbits. By combining this same technique with numerical simulations, it was shown in COS01] that one obtains similar results when introducing a periodic forcing to a two-dimensional integrate-and-fire-orbursting system. There authors studied in more detail the existence of periodic orbits (mode locking) and showed numerically that the firing number may follow a devil's staircase as a function of parameters.

In this work we present a new approach to study periodically forced systems with resets based on the use of the so-called stroboscopic map (time- $T$ return 
map). One of the advantages of our approach is that it leads to a general setting for periodically forced systems. Already in the smooth case such systems are better understood by means of the stroboscopic map than with Poincaré maps onto transverse sections in the state space. In the latter, as mentioned above, one has to check for congruency between the passage times and the period of the forcing, which requires explicit knowledge of the solutions of the system or approximations by circle maps in order to study the existence of periodic orbits. However, when dealing the with stroboscopic map, this is reduced to the study of existence of fixed (or periodic) points of this map, which comes by applications of classical results as the implicit function theorem (when studying perturbations) or Brouwer's theorem.

The use of the stroboscopic maps has been avoided in systems with resets because it becomes a discontinuous (piecewise-smooth) map. The sets of initial condition for which this map becomes smooth are identified by the number of spikes exhibited by their trajectories when flowed for a time $T$, and hence it is potentially defined in an arbitrarily large number of partitions. However, in this work we use recent results in non-smooth systems to face these discontinuities and rigorously study a general and large class of hybrid systems with resets. More precisely, we consider a one-dimensional monotonic system with an attracting subthreshold equilibrium point under a $T$-periodic forcing consisting of a periodic square wave function. This is given by a pulse of amplitude $A$ and duration $d T$, with $0 \leq d \leq 1$ (duty cycle), and is 0 for the rest of the period $T$. Such a periodic stimulus is used in many contexts, as the stimulation of an excitable cell (e.g., an hormone release) frequently occurs in a pulsatile form. Such a periodic forcing adds indeed an extra non-smoothness, as one switches from one autonomous system to another one at the switching times, when the pulse is switched on or off. However, this singularity consists of a discontinuity on the field that does not add nor remove extra dynamical objects nor bifurcations. As usual when dealing with piecewise-smooth fields, in this case the solutions of the system are continuous and obtained by proper concatenation of the solutions of the both fields, as long as the threshold is not reached.

Our results consist of a rigorous and detailed description of the bifurcation scenario formed by the parameters describing the input pulse: its amplitude $A$ and its duty cycle $d$. We show that there exists an infinite number of regions in this two-dimensional parameter space for which the stroboscopic map exhibits a fixed point. The regions in between are covered by regions of existence periodic orbits following the so-called period adding structure. The rotation number associated with these periodic orbits is a devil's staircase as a function of the parameters. This allows us to show that the asymptotic firing rate of the system also follows a devil's staircase when the mentioned parameters are varied.

This work is organized as follows.

In section 2 we describe the system, announce our results and describe the state of the art in piecewise-smooth maps relevant for our work. In section 3 we prove our results, and in section 4 we validate them by providing numerical computations for three examples. 


\section{$2 \quad$ Background and results}

Let us consider a non-autonomous periodic system given by

$$
\dot{x}=f(x)+I(t), x \in \mathbb{R}
$$

with $f(x) \in C^{\infty}(\mathbb{R})$ and $I(t)$ a $T$-periodic square-wave function

$$
I(t)= \begin{cases}A & \text { if } t \in(n T, n T+d T] \\ 0 & \text { if } t \in(n T+d T,(n+1) T],\end{cases}
$$

with $0 \leq d \leq 1$. Let us submit system 2.1 to the reset condition

$$
x=\theta \longrightarrow x=0 ;
$$

that is, the trajectories of system 2.1 are instantaneously reset to 0 whenever they reach the threshold given by $x=\theta$. This provides a new system which is in the class of hybrid systems, as it combines an algebraic condition with a differential equation. Due to the generality of the function $f$, this system represents a large class of the spiking models which are used as simplification of slow-fast systems modeling excitable cells as neurons. Hence, we will refer to the discontinuities given by the reset condition as spikes.

Let us assume that the system

$$
\dot{x}=f(x)
$$

satisfies the following conditions

H.1 it possesses an attracting equilibrium point

$$
0<\bar{x}<\theta,
$$

H.2 $f(x)$ is monotonic decreasing function in $[0, \theta]$ :

$$
f^{\prime}(x)<0,0 \leq x \leq \theta .
$$

The reset condition 2.3), in combination with conditions H.1-H.2, implies that the dynamics we are interested in is located in the interval $[0, \theta)$. In fact, by identifying $0 \sim \theta$, this interval can be seen as a circle. However, for our convenience, we will consider from now on that the state space will be given by the interval $[0, \theta)$.

Note that the fact that the equilibrium point $\bar{x}$ is globally attracting in $[0, \theta)$ prevents the system to exhibit spikes without the input. It can hence be considered as the limit of a slow/fast system. 


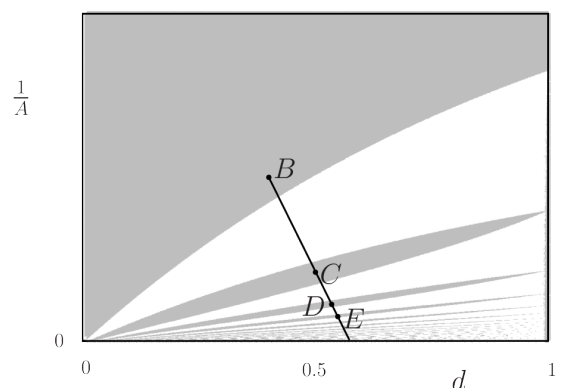

(a)

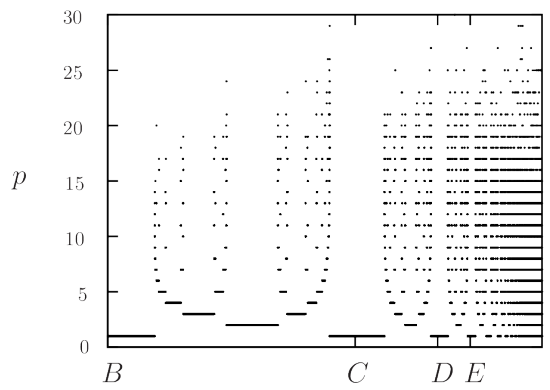

(b)

Figure 1: (a) Bifurcation scenario for system (2.1)-(2.3). In gray regions there exist $T$-periodic orbits, in white ones higher periodic orbits following and adding structure (see text). In $B, C, D$ and $E$ one finds $T$-periodic orbits spiking 0,1 , 2 and 3 times per period, respectively. (b) periods of the periodic orbits found along the line shown in (a).

One of the most relevant features of the asymptotic dynamics of system (2.1)2.3) is the firing rate, i.e. the average number of spikes exhibited by the system per unit time:

$$
r\left(x_{0}\right)=\lim _{\tau \rightarrow \infty} \frac{\#\left(\text { spikes performed by } \phi\left(t ; x_{0}\right) \text { for } t \in[0, \tau]\right)}{\tau},
$$

where $\phi\left(t ; x_{0}\right)$ is a trajectory of the system with $\phi\left(0 ; x_{0}\right)$. When the system possesses an attracting periodic orbit, this quantity does not depend on the initial condition $x_{0}$ and becomes the number of spikes performed by this periodic orbit along one period divided by the length of the period.

Hence, to obtain qualitative and quantitative description of the firing rate we study the existence of periodic orbits for system (2.1)-(2.3) and their bifurcations under conditions H.1-H.2. In particular, we provide a full description of the bifurcation scenario for the two-dimensional parameter space $d \times 1 / A$, which is shown in fig. 1. More precisely, under conditions H.1-H.2 and for any $T>0$, we prove the following.

- The parameter space $d \times 1 / A, d \in(0,1)$, contains an infinite number of disjoint bounded regions (gray regions in fig. 1(a) for which the system (2.1)(2.3) possesses a unique globally attracting $T$-periodic orbit (fixed point of the stroboscopic map). These regions are clockwise ordered in such a way that the $T$-periodic orbit corresponding to the next region exhibits one spike more per period than the previous one (propositions 3.2 and 3.3 . 
- In between the regions corresponding to the existence of fixed points of the stroboscopic map (grey regions) are the regions defined by the existence of periodic orbits of arbitrarily large periods (white regions). These periodic orbits are organized by the period adding structure (proposition 3.4.

- Along any invertible and continuous curve

$$
\lambda \in \mathbb{R} \longmapsto(d(\lambda), 1 / A(\lambda))
$$

satisfying $d(\lambda) \in(0,1),(1 / A(\lambda))^{\prime}<0,1 / A(\lambda) \rightarrow 0$ when $\lambda \rightarrow \infty$ and crossing all the regions mentioned above (as the straight line labeled in fig. 1(a) , the firing rate evolves following a devil's staircase from 0 to $\infty$. This is explained in section 3.4 and is a consequence of proposition 3.4 .

The main tool that we will use to prove the previous rsults is the stroboscopic map at return time $T$. As it will be shown, this is a piecewise-smooth map defined on an infinite number of domains containing initial conditions for which the system (2.1)-(2.3) exhibits different number of spikes when flowed for a time $T$. For each domain, there exist parameter values $d$ and $A$ for which this map has a fixed point located in this domain; these parameter values correspond to the gray regions in fig. 1 .

Between two consecutive regions where fixed points exist (white regions in fig. 1(a), the stroboscopic map is discontinuous at the boundary between two consecutive domains, and can be written as the normal form map

$$
g(x)= \begin{cases}\mu_{\mathcal{L}}+g_{\mathcal{L}}(x) & \text { if } x<0 \\ -\mu_{\mathcal{R}}+g_{\mathcal{R}}(x) & \text { if } x>0,\end{cases}
$$

with $g_{\mathcal{L}}, g_{\mathcal{R}}$ smooth functions satisfying $g_{\mathcal{L}}(0)=g_{\mathcal{R}}(0)=0$. As the stroboscopic map is given by the integration of a flow, its fixed points have positive associated eigenvalues and hence $g_{\mathcal{L}}(x)$ and $g_{\mathcal{R}}(x)$ are increasing functions near the origin. Moreover, due to the assumption $f^{\prime}<0$, the flow is everywhere contracting, so that $\left|g_{\mathcal{L}}^{\prime}\right|<1$ and $\left|g_{\mathcal{R}}^{\prime}\right|<1$.

We now describe the bifurcation scenario for the map (2.7), which is shown in fig. 2

Note that, for $\left|\mu_{\mathcal{L}}\right|,\left|\mu_{\mathcal{R}}\right|$ small enough, if $\mu_{\mathcal{L}}<0$ and $\mu_{\mathcal{R}}<0$ the map (2.7) possesses two fixed points, each at each side of the boundary $x=0$, which undergo border collision bifurcations when $\mu_{\mathcal{L}}=0$ and $\mu_{\mathcal{R}}=0$, respectively. Hence, for $\mu_{\mathcal{L}}=\mu_{\mathcal{R}}=0$ both fixed points simultaneously collide with the boundary, undergo border collision bifurcations and no longer exist. For $\mu_{\mathcal{L}}, \mu_{\mathcal{R}}>0$ there exist an infinite number of border collision bifurcation curves separating regions of existence of periodic orbits with arbitrarily high periods. These are organized by the so-called period adding phenomenon. To explain this, we first introduce the usual symbolic dynamics.

Let us assume that $\left(x_{1}, . ., x_{n}\right)$ is a $n$-periodic orbit of the map (2.7). To this periodic orbit we assign a symbolic encoding given by

$$
\begin{array}{ll}
x_{i} \rightarrow \mathcal{L} & \text { if } x_{i}<0 \\
x_{i} \rightarrow \mathcal{R} & \text { if } x_{i}>0 .
\end{array}
$$




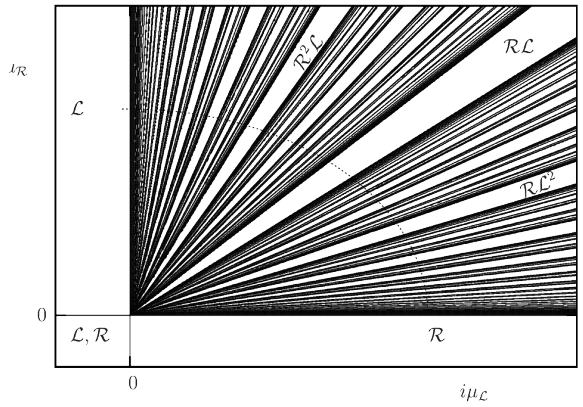

(a)

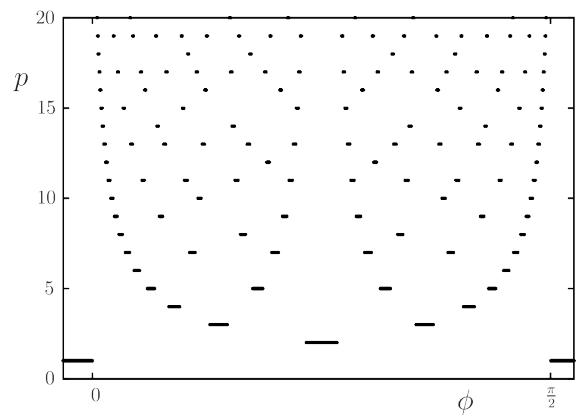

(b)

Figure 2: The period adding big bang or gluying bifurcation. (a) $\mu_{\mathcal{L}} \times \mu_{\mathcal{R}}$ parameter space, (b) periods of the periodic orbits along the curve shown in (a).

For each periodic orbit we obtain a symbolic sequence of $\mathcal{L}$ 's and $\mathcal{R}$ 's depending on whether the orbit steps on the left or on the right of the boundary. Obviously, the length of the symbolic sequence is the period of the periodic orbit.

Then, the period adding structure is described as follows. Between the regions of existence of periodic orbits with symbolic sequences $\sigma_{1}$ and $\sigma_{2}$ and periods $n_{\sigma_{1}}$ and $n_{\sigma_{2}}$ there exists a region corresponding to the existence of a periodic orbit with symbolic sequence $\sigma_{1} \sigma_{2}$. Clearly, the period of this periodic orbit is $n_{\sigma_{1}}+n_{\sigma_{1}}$. This is occurs ad infinitum as illustrated in fig. 2(b), where one can see the periods of the periodic orbits obtained when crossing all these regions along a curve as the one shown in fig. 2(a).

Beyond the evolution of the periods of the periodic orbits, one of the most relevant quantities associated with the adding scheme is the rotation number corresponding to the periodic orbits obtained when crossing all these regions in the parameter space. As shown in fig. 3(a), the rotation number associated to each periodic orbit is obtained by the dividing the number of $\mathcal{R}$ symbols contained in its symbolic sequence by the period of the periodic orbit (see GGT84, GIT84). Hence, the rotation number follows a devil's staircase from 0 to 1 when parameters are varied along curves as the one shown in fig.2(a). A devil's staircase is a monotonically increasing continuous function which is constant almost everywhere, except for a Cantor set of zero measure. In this Cantor set, which consists of the border collision bifurcation curves shown in fig. 2(a) the rotation number becomes irrational and no periodic orbit exists but a quasi-periodic one.

The period adding phenomenon described above was first described in [GGT84, and later studied in more detail in GPTT86, LPZ89, TS86, PTT87, Gam87, GT88. Subsequently, this was rediscovered in AS06 when numeri- 


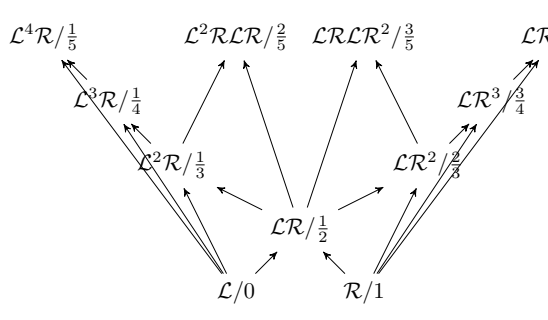

(a)

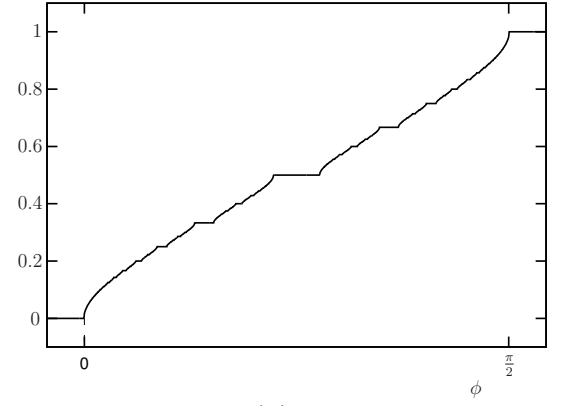

(b)

Figure 3: (a) Symbolic sequences and rotation numbers forming a Farey tree structure for the period adding. (b) Devil's staircase associated with the rotation numbers along the Farey tree, obtained obtained along a curve as the one labeled in fig. 2(a).

cally investigating the map 2.7) with $g_{\mathcal{L}}$ and $g_{\mathcal{R}}$ linear functions. The authors of [AS06] provided an accurate description of the bifurcation scenario in terms of non-smooth language (border collision bifurcations). A special interest was given to the codimension-two bifurcation at the origin of the $\mu_{\mathcal{L}} \times \mu_{\mathcal{R}}$ parameter space, which was called period-adding big bang bifurcation, to distinguish it from the so-called period-incrementing big bang bifurcation, being both a special type of the gluing bifurcation reported in CGT84, GGT88. The period incrementing case, which exhibits a completely different bifurcation scenario, occurs when one of the functions $g_{\mathcal{L}}$ or $g_{\mathcal{R}}$ is decreasing and the other one increasing near the origin (being both contracting), as was rigorously proven in AGS11. The result in the adding case (relevant to this paper) is stated below. A complete proof of this result has not been published, but it can be pieced together from partial results presented in Gam87, GT88, GGT88.

Theorem 2.1. Let $g$ be a map as in eq. 2.7. such that $g_{\mathcal{L}}$ and $g_{\mathcal{R}}$ are increasing contracting functions,

$$
\begin{array}{ll}
0<\left(g_{\mathcal{L}}(x)\right)^{\prime}<1 & x \in(-\infty, 0) \\
0<\left(g_{\mathcal{R}}(x)\right)^{\prime}<1 & x \in(0, \infty)
\end{array}
$$

Consider a $C^{1}$ curve in the parameter space $\mu_{\mathcal{L}} \times \mu_{\mathcal{R}}$

$$
\begin{array}{rlc}
\gamma:[0,1] & \longrightarrow & \mathbb{R}^{2} \\
\lambda & \longmapsto & \left(\mu_{\mathcal{L}}(\lambda), \mu_{\mathcal{R}}(\lambda)\right)
\end{array}
$$

satisfying 
$C .1 \mu_{\mathcal{L}}(\lambda)>0$ and $\mu_{\mathcal{R}}(\lambda)>0$ for $\lambda \in(0,1)$

C.2 $\left(\mu_{\mathcal{L}}(\lambda)\right)^{\prime}>0$ and $\left(\mu_{\mathcal{R}}(\lambda)\right)^{\prime}<0$ for $\lambda \in[0,1]$

C.3 $\mu_{\mathcal{L}}(0)=0, \mu_{\mathcal{R}}(1)=0$

Then, the bifurcation diagram exhibited by the map $g_{\lambda}$ obtained from eq. 2.7) after performing the reparametrization given by $\gamma$, follows a period adding strucutre for $\lambda \in[0,1]$. Moreover, the rotation number $\rho\left(g_{\lambda}\right)$ of the map $g_{\lambda}$ is a devil's staircase as a function of $\lambda$ such that $\rho\left(g_{0}\right)=0$ and $\rho\left(g_{1}\right)=1$.

Remark 2.1. The increasing and contractive condition 2.9 can be relaxed to be satisfied locally near $x=0$ if $\mu_{\mathcal{L}}(\lambda)$ and $\mu_{\mathcal{R}}(\lambda)$ are small enough for $\lambda \in(0,1)$. This is because the periodic orbits are located in the aboserving interval $\left[-\mu_{\mathcal{R}}(\lambda), \mu_{\mathcal{L}}(\lambda)\right]$.

By means of theorem 2.1 we will show that the stroboscopic map follows period adding bifurcation structures when parameters are varied along curves as the straight line shown in fig. 1(a) the white regions in fig. 1 1 surrounded by gray ones are filled by the bifurcation structres shown in fig. 2. We will use the properties of the devil's staircase of the rotation number to deduce properties of the firing rate defined in eq. 2.6p.

\section{Dynamics of the stroboscopic map}

\subsection{Notation, properties and subthreshold dynamics}

Let

$$
\varphi\left(t ; x_{0} ; A\right)
$$

be the flow associated with system

$$
\dot{x}=f(x)+A
$$

such that $\varphi\left(0 ; x_{0} ; A\right)=x_{0}$. As usual in piecewise-defined systems, by properly concatenating the flows $\varphi\left(t ; x_{0} ; A\right), \varphi\left(t ; x_{0} ; 0\right)$ and the reset condition 2.3 one obtains the flow

$$
\phi\left(t ; x_{0}\right)
$$

associated with system (2.1)-2.3 such that $\phi\left(0 ; x_{0}\right)=x_{0}$. Due to the reset conditions this flow is discontinuous at the times for which spikes occur. In addition, due to the discontinuities of the forcing $I(t)$ defined in 2.2 , the flow $\phi$ is non differentiable at $t=d T \bmod T$.

Note that for the definition of the flow $\phi$ associated with the non-autonomous system (2.1) we consider that the initial condition $x_{0}$ occurs at $t_{0}=0$. This will be assumed in the rest of this work and, as we are interested on the existence of periodic orbits, does not present any loss generality. 
Under assumptions H.1-H.2 we first study the subthreshold dynamics; that is, invariant objects of system (2.1)-2.3) that do not interact with the threshold (do not exhibit spikes).

On one hand, if $d=0$, then $I(t)=0$ and the system (2.1)- 2.3 has the same dynamics as the autonomous system (2.4). That is, it has an attracting equilibrium point at $x=\bar{x}$.

On the other hand, if $d=1$, then $I(t)=A$. Hence system 2.1)-2.3 becomes the autonomous system (3.2) subject to the reset condition (2.3) and, as for $d=0$, the $T$-periodic forcing does not play any role. By the implicit function theorem, if $A>0$ is small enough the system possesses an attracting equilibrium point at

$$
x^{*}=\bar{x}-\frac{A}{f^{\prime}(\bar{x})}+O\left(A^{2}\right)<\theta .
$$

Note that, as $f(x)$ is a monotonically decreasing function (condition H.2,) and hence this equilibrium point increases with $A$ towards the boundary $x=\theta$. Thus, for $d=1$, when $A$ is large enough this equilibrium point collides with the boundary $x=\theta$, undergoes a border collision bifurcation. After that, the system has a periodic orbit that spikes once per period. The period of this periodic orbit, $\delta>0$, becomes the time needed by system $\dot{x}=f(x)+A$ with initial condition $x_{0}=0$ to reach the threshold $x=\theta$. This is the smallest $\delta>0$ such that

$$
\varphi(\delta ; 0 ; A)=\theta,
$$

if it exists ( $A$ is large enough).

Let us now study the subthreshold dynamics of the system when $0<d<1$. We have the next

Proposition 3.1. Let

$$
Q:=\frac{1}{T} \int_{0}^{T} I(t) d t=A d
$$

be small enough. Then, if $T>0$ is small enough, system 2.1-2.3) has a $T$-periodic orbit that does not hit the boundary $x=\theta$.

Proof. By averaging the system. After the time rescale $\tau=\frac{t}{T}$ system 2.1 becomes

$$
\dot{x}=T(f(x)+I(\tau T)),
$$

where "means now derivative with respect to $\tau$ and $I(\tau T)$ is 1-periodic. We now consider the averaged version of system (3.5),

$$
\dot{y}=T(f(x)+Q) .
$$

By the averaging theorem, as system 3.5 is Lipschitz in $x$, if system 3.6 possesses a hyperbolic equilibrium point then, for $T>0$ small enough, system (3.5) possesses a hyperbolic periodic orbit (see [BM61, GH83]). This will 
occur if $Q>0$ is small enough, and the equilibrium point of $(3.6)$ will be of the form

$$
\bar{y}=\bar{x}-\frac{Q}{f^{\prime}(\bar{x})}+O\left(Q^{2}\right)<\theta,
$$

where $\bar{x}$ is the hyperbolic equilibrium point of system (2.4).

Then, the periodic orbit of system (3.5) will be $T$-close to $\bar{y}$.

When $A$ is large, the periodic orbit given by the previous lemma may undergo a border collision bifurcation and lead to spiking dynamics. This new dynamics will be studied in sections 3.2 and 3.3 by means of the stroboscopic map, i.e. the time- $T$ return map

$$
\begin{array}{rlc}
\mathfrak{s}:[0, \theta) & \longrightarrow & {[0, \theta)} \\
x_{0} & \longmapsto \phi\left(T ; x_{0}\right) .
\end{array}
$$

The main results in this work rely on the fact that $\mathfrak{s}$ is a piece-smooth map. To see this, we define the sets

$$
\begin{gathered}
S_{n}=\left\{x_{0} \in[0, \theta) \text { s.t. } \phi\left(t ; x_{0}\right) \text { reaches the threshold }\{x=\theta\}\right. \\
n \text { times for } 0 \leq t \leq T\}, n \geq 0 .
\end{gathered}
$$

When restricted to some set $S_{n}$, the flow $\phi\left(t ; x_{0}\right)$ becomes a certain combination of the smooth flows $\varphi\left(t ; x_{0} ; A\right), \varphi\left(t ; x_{0} ; 0\right)$ and the smooth mapping $\theta \mapsto 0$ (given by the reset condition) which does not depend on the initial condition $x_{0} \in S_{n}$. Hence, the stroboscopic map $\mathfrak{s}$ becomes a smooth map in each of the sets $S_{n}$ and is as regular as the flow $\varphi(t ; A)$. Given $m \neq n$, the flow, when restricted to $S_{n}$ and $S_{m}$, performs a different number of spikes in the time window $[0, T]$. Hence $\mathfrak{s}$ is given by a different combination of the mentioned maps. Thus, $\mathfrak{s}$ is smooth on the interiors of $S_{n}$ and $S_{m}$ but will typically be discontinuous at its boundaries.

Note that some of the sets $S_{n}$ may be empty. The next lemma tells us that at most two of them are non empty and they must be consecutive (see fig. 4 for $S_{2}$ and $S_{3}$ ). Moreover, let $\Sigma_{n} \in S_{n}$ be defined by the requirement

$$
\phi\left(d T ; \Sigma_{n}\right)=\theta .
$$

That is, the (unique) initial condition whose trajectory spikes $n$ times and performs its last spike precisely at $t=d T$. The next lemma tells us also that $\Sigma_{n}$ is the value at which the map $\mathfrak{s}$ exhibits a discontinuity and separates the sets $S_{n-1}$ and $S_{n}$.

Lemma 3.1. Assume that there exists $\Sigma_{n} \in S_{n}$ as given in eq. (3.9) for some $n \geq 1$. Then the following statements hold:

i) If $\Sigma_{i}, \Sigma_{j} \in[0, \theta)$, then $i=j$.

ii) If $\Sigma_{n} \in(0, \theta)$, then $\left[0, \Sigma_{n}\right)=S_{n-1}$ and $\left[\Sigma_{n}, \theta\right)=S_{n}$. 


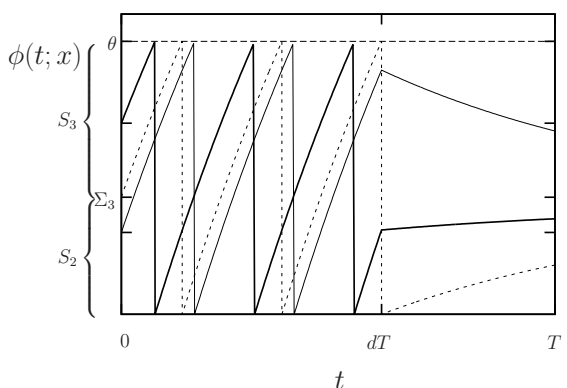

(a)

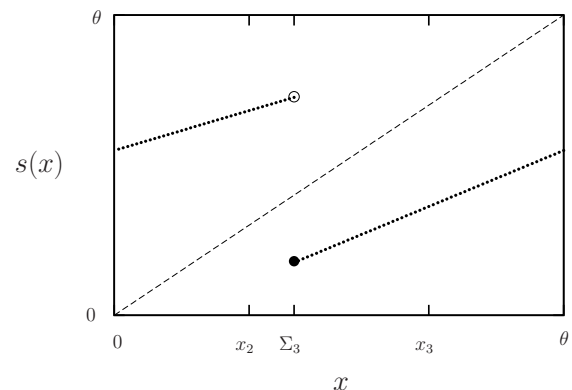

(b)

Figure 4: In (a) the trajectories of systems (2.1)-(2.3). Dashed line: trajectory with $\Sigma_{3}$ as initial condition. Thick line: trajectory with $x_{3}>\Sigma_{3}$ as initial condition, which spikes 3 times. Normal line: trajectory with $x_{2}<\Sigma_{3}$ as initial condition, which spikes 2 times. In (b) the stroboscopic map, with a discontinuity at $x=\Sigma_{3}$.

iii) If $\Sigma_{n}=0$, then $[0, \theta)=S_{n}$.

Proof. The first part $i$ ) comes from the fact that the flow $\phi$ is invertible. Hence, if it exists, the initial condition that makes $\phi$ reach $\theta$ at $t=d T$ is unique.

We now see $i i$ ). Let

$$
t_{n}<t_{n}+\delta<\cdots<t_{n}+(n-2) \delta<t_{n}+(n-1) \delta=d T
$$

be the sequence of times for which the trajectory $\phi\left(t ; \Sigma_{n}\right)$ exhibits spikes. Let us now take an initial condition $x_{0}<\Sigma_{n}$. For $t<d T$ the flow $\phi\left(t ; x_{0}\right)$ becomes the flow of the autonomous system $\dot{x}=f(x)+A$ plus the reset condition (2.3). Hence, the first spike of the trajectory $\phi\left(t ; x_{0}\right)$ occurs for $t>t_{n}$. By induction, the $n$th spike for such a trajectory occurs for $t \in(d T-\delta, d T)$, with $\delta$ as in eq. (3.4). For $t>d T$ the trajectory $\phi\left(t ; x_{0}\right)$ becomes the flow of system $\dot{x}=f(x)$, which has an attractor $\bar{x} \in(0, \theta)$; hence, no other spikes can occur for $t>d T$ and the trajectory $\phi\left(t ; x_{0}\right)$ exhibits exactly $n-1$ spikes for $0 \leq t \leq T$. Using $i$ ) we get that, $\left[0, \Sigma_{n}\right)=S_{n-1}$. Arguing similarly, any trajectory $\phi\left(t ; x_{0}\right)$ with $x_{0} \in\left[\Sigma_{n}, \theta\right)$ exhibits exactly $n$ spikes for $0 \leq t \leq T$ and thus $\left[\Sigma_{n}, \theta\right)=S_{n}$. Proceeding similarly, if $\Sigma_{n}=0$, then all initial conditions in $[0, \theta)$ lead to trajectories exhibiting a $n$th spike for $t \in(d T-\delta, d T)$. Hence, $S_{n}=[0, \theta)$.

Remark 3.1. The values $\Sigma_{n}$ become upper and lower boundaries of the sets $S_{n-1}$ and $S_{n}$, respectively. 
The following results give us properties of the boundaries $\Sigma_{n}$ which we will use in section 3.2 .

Lemma 3.2. Let $d \in(0,1), A>0$ and assume $\Sigma_{n} \in(0, \theta)$. Then $\Sigma_{n}$ is a monotonically decreasing function of $A$.

Proof. To avoid confusions with the differential and derivative, in this proof we rename the duty cycle $d$ by $a$.

The boundary $\Sigma_{n}$ is determined by the initial condition such that the flow $\phi\left(t ; x_{0}\right)$ collides with the boundary for the $n$th time at $t=a T$. Let $\varphi\left(t ; x_{0} ; A\right)$ be the flow defined in eq. (3.1), and let $\delta>0$ be the time needed by with initial condition $x_{0}=0$ to reach the threshold $\theta$, as defined in eq. (3.4). Note that $\delta$ is a decreasing function of $A$. In fact, rewriting eq. (3.4) as

$$
\int_{0}^{\theta} \frac{d x}{f(x)+A}=\delta
$$

we get that

$$
\frac{d \delta}{d A}=-\int_{0}^{\theta} \frac{d x}{(f(x)+A)^{2}}<0 .
$$

The boundary $\Sigma_{n}$ is thus determined by the equation

$$
\varphi\left(a T-(n-1) \delta ; \Sigma_{n}, A\right)=\theta,
$$

or, equivalently, by the equation

$$
\int_{\Sigma_{n}}^{\theta} \frac{d x}{f(x)+A}=a T-(n-1) \delta
$$

Differentiating we get that

$$
\frac{d \Sigma_{n}}{d A}=-\left(f\left(\Sigma_{n}\right)+A\right)\left(\int_{\Sigma_{n}}^{\theta} \frac{d x}{(f(x)+A)^{2}}-(n-1) \frac{d \delta}{d A}\right) .
$$

Noting that $f\left(\Sigma_{n}\right)+A>0$ because the system $\dot{x}=f(x)+A$ possesses an attracting equilibrium point for $x>\theta$ (which permits to spike), we get that

$$
\frac{d \Sigma_{n}}{d A}<0
$$

The next lemma provides the lateral values of the stroboscopic map $\mathfrak{s}$ at the discontinuities.

Lemma 3.3. Let

$$
\begin{aligned}
& \mathfrak{s}_{-}=\varphi(T(1-d) ; \theta ; 0) \\
& \mathfrak{s}_{+}=\varphi(T(1-d) ; 0 ; 0),
\end{aligned}
$$


where $\varphi\left(t ; x_{0} ; A\right)$ is the flow defined in (3.1).

If $\Sigma_{n} \in[0, \theta)$, then the lateral images of $\Sigma_{n}$ by $\mathfrak{s}$ do not depend on $A$ or $n$ and become

$$
\begin{gathered}
\mathfrak{s}\left(\Sigma_{n}^{-}\right)=\liminf _{x \rightarrow \Sigma_{n}} \mathfrak{s}(x)=\mathfrak{s}_{-} \\
\mathfrak{s}\left(\Sigma_{n}^{+}\right)=\limsup _{x \rightarrow \Sigma_{n}} \mathfrak{s}(x)=\mathfrak{s}_{+},
\end{gathered}
$$

Proof. The trajectory of $\Sigma_{n}$ is such that the flow $\phi\left(t ; \Sigma_{n}\right)$ reaches the threshold for the $n$th time exactly at $t=d T$. The limiting value $\mathfrak{s}\left(\Sigma_{n}^{-}\right)$is obtained assuming that the flow does not spike at $t=d T$ (the threshold is almost reached). Hence, one just needs to integrate the flow $\varphi\left(t ; x_{0} ; 0\right)$ from $t=d T$ to $t=T$ with initial condition $x_{0}=\theta$. This gives eq. (3.13).

On the other hand, the right image is computed adding a new spike at $t=d T$. Hence, one has only to proceed identically but replacing the initial condition by the one given by the reset, $x_{0}=0$.

\subsection{Border collision bifurcations of spiking fixed points}

As noted in the previous section, non spiking periodic orbits (without interaction with the threshold) exist for $A>0$ small enough and $d=0$ or $d=1$. Hence, the natural parameter space where to study the possible bifurcations leading to spiking dynamics is $d \times A$. Equivalently, we will consider $d \times 1 / A$ instead. This is because, in this space, the bifurcation curves will be bounded.

In order to show that the bifurcation scenario in the parameter space $d \times 1 / A$ for system 2.1)-(2.3) is equivalent to the one shown in fig. 1 and described in $\$ 2$, we first show the existence of an infinite number of regions for which $\mathfrak{s}$ possesses fixed points. These fixed points are located at different domains $S_{n}$ defined in (3.8). These regions in the parameter space are the gray regions in fig. 1 . As shown in the next result, when they exist, these fixed points are unique and are located at one side of some discontinuity $\Sigma_{n}$. By varying parameter values, they bifurcate when entering the white regions in figure $1(\mathrm{a})$.

The next proposition provides the existence of unique fixed points, $\bar{x}_{n} \in S_{n}$ for any $n \geq 0$.

Proposition 3.2. Let $d \in(0,1)$ and $n \geq 0$. Then, there exists some value of A for which the stroboscopic map $\mathfrak{s}$ defined in (3.7) possesses a fixed point $\bar{x}_{n} \in S_{n}$. When it exists, this fixed point becomes the only invariant object of $\mathfrak{s}$.

Proof. The existence of the fixed point $\bar{x}_{0} \in S_{0}$ of the stroboscopic map $\mathfrak{s}$ (3.7) for any $d \in(0,1)$ when $A>0$ is small enough comes from proposition 3.1 By induction we now show that, for any $n \geq 0$, there exist values of $A$ for which one finds fixed points of $\mathfrak{s}, \bar{x}_{n}$, leading to periodic orbits spiking $n$ times: $\bar{x}_{n} \in S_{n}$. Assume that there exists a fixed point $\bar{x}_{n-1} \in S_{n-1}$. By if necessary increasing $A$, we can assume that $S_{n-1}=\left[0, \Sigma_{n}\right)\left(\bar{x}_{n-1}\right.$ is located at the left of the discontinuity). Then, by lemma $3.2, \Sigma_{n}$ is a monotonically decreasing 


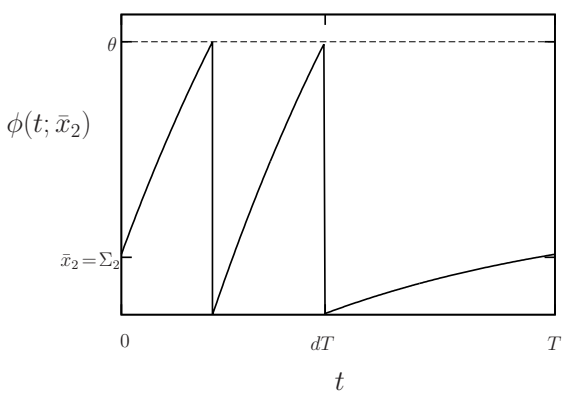

(a)

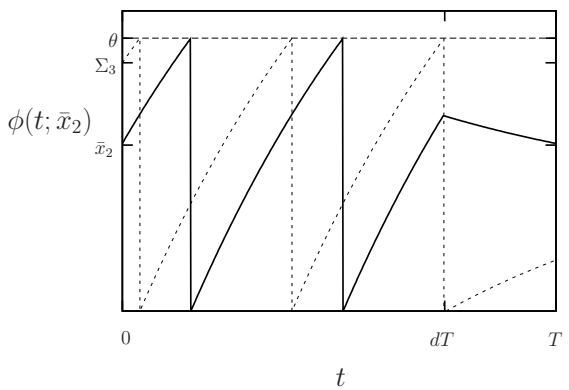

(c)

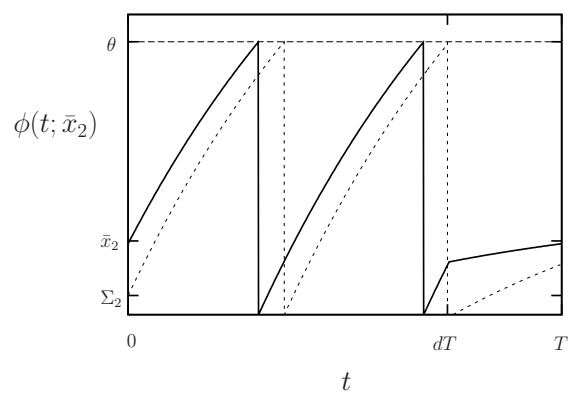

(b)

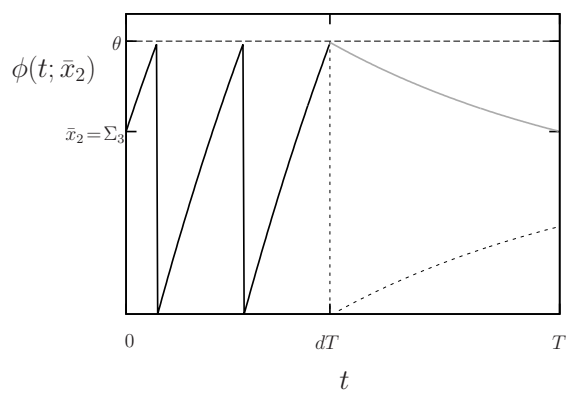

(d)

Figure 5: $T$-periodic orbit spiking twice per period (fixed point $\bar{x}_{2}$ of the stroboscopic map) a its bifurcations when $A$ is varied along $\left(A_{2}^{\mathcal{R}}(d), A_{2}^{\mathcal{L}}(d)\right)$. It undergoes border collision bifurcations when it collides with the boundaries $\Sigma_{2}$ and $\Sigma_{3}$ (a) and (d), respectively. The periodic orbit shown in (d) is its limit when $\bar{x}_{2} \rightarrow \Sigma_{3}^{-}$; note that for $\bar{x}_{2}=\Sigma_{3}$ it should be reset to 0 at $t=d T$, this is why it is shown in gray. In (b) and (c), the trajectories of these boundaries are shown in dashed lines; note that they collide with the threshold at $t=d T$. Parameter values for panel (c) are the same as for point $D$ of figure 1(a). The four figures are a in one to one correspondence with the four figures of figure 6 . where the stroboscopic map is shown. 


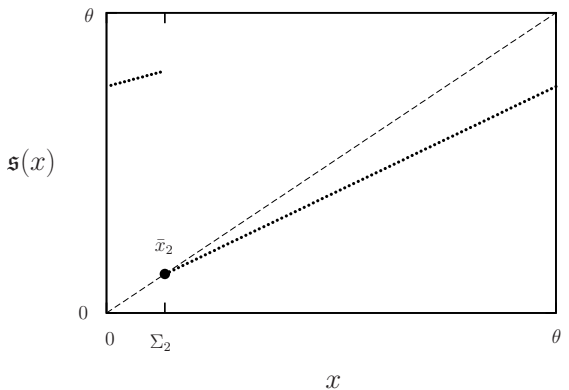

(a)

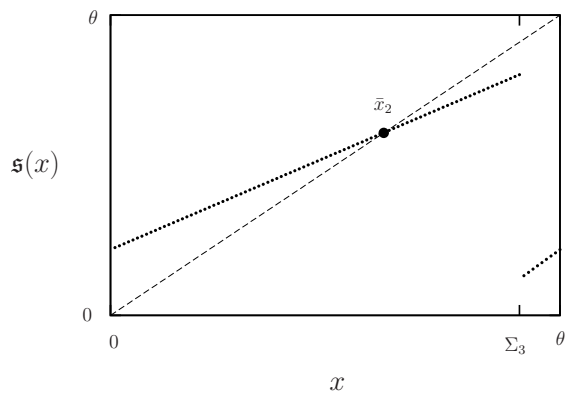

(c)

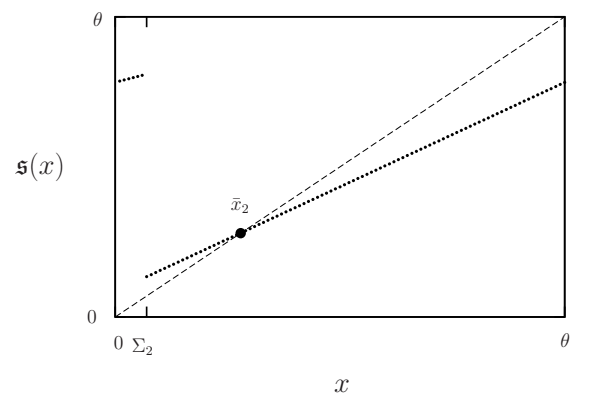

(b)

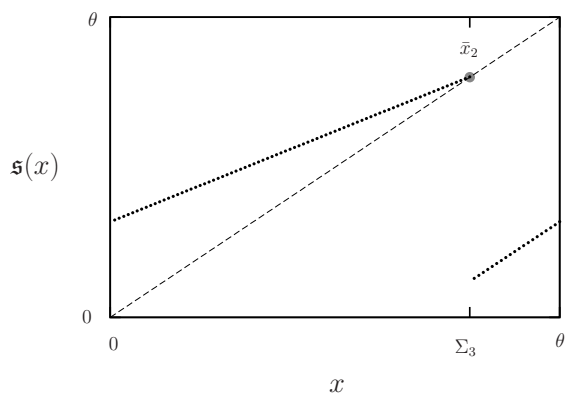

(d)

Figure 6: Stroboscopic map for the $T$-periodic orbits shown in figure 5 . In (a) and (d) the fixed point $\bar{x}_{2}$ undergoes border collision bifurcation when it collides with the boundaries $\Sigma_{2}$ from the right and $\Sigma_{3}$ from the left, respectively. Note that, in (d) the fixed point is shown in gray to emphasize that the map takes indeed the value on the right for $x=\Sigma_{3}$. In (b)-(c) the boundary $\Sigma_{2}$ disappears and a new boundary $\Sigma_{3}$ appears while the fixed point $\bar{x}_{2}$ remains. 
function of $A$. Hence, by further increasing $A$, one necessary finds some value, $A=A_{n}^{\mathcal{C}}(d)$ for which $\Sigma_{n}=0$. At this point, by lemma 3.1 iii), we get that $[0, \theta)=S_{n}$ and the map $\mathfrak{s}$ becomes continuous. Hence, as $\mathfrak{s}\left(S_{n}\right) \subset S_{n}$ and $\mathfrak{s}$ is a contracting map, there necessary exists a fixed point $\bar{x}_{n} \in S_{n}$ for $A=A_{n}^{\mathcal{C}}(d)$.

We now show that, when they exist, the fixed points $\bar{x}_{n}$ are the only possible invariant objects. On one hand note that the monotonicity of $f(x)$ and the stability of $\bar{x} \in(0, \theta)$ imply that $\mathfrak{s}$ is monotonically increasing and contracting in $S_{n}$. On the other one we recall that, from lemma 3.1, we use that at most two sets $S_{n-1}$ and $S_{n}$ or $S_{n}$ and $S_{n+1}$ can coexist. Hence, if $\mathfrak{s}$ possesses a fixed point $\bar{x}_{n} \in S_{n}$, there exist only three possible situations:

1. $\bar{x}_{n} \in(0, \theta) \subset S_{n}$

2. $\left[0, \Sigma_{n}\right)=S_{n-1}$ and $\bar{x}_{n} \in\left[\Sigma_{n}, \theta\right)=S_{n}$

3. $\bar{x}_{n} \in\left(0, \Sigma_{n+1}\right) \subset S_{n}$ and $\left[\Sigma_{n+1}, \theta\right)=S_{n+1}$

Note that $\bar{x} \in(0, \theta)$ implies that necessary $\bar{x}_{n} \neq 0$.

In the first case no other periodic points are possible because $\mathfrak{s}$ is monotonically increasing, contracting and continuous in all its domain.

In the second and third cases this becomes a direct consequence of the fact that $\mathfrak{s}$ has a negative gap at its discontinuities (illustrated in fig. 6); that is,

$$
\lim _{x \rightarrow\left(\Sigma_{n}\right)^{-}} \mathfrak{s}(x)>\lim _{x \rightarrow\left(\Sigma_{n}\right)^{+}} \mathfrak{s}(x), \forall n \geq 0,
$$

(see fig. 4 for $n=3$ ). This is a direct consequence of lemma 3.3 .

The next proposition provides us the bifurcation curves at which the fixed points $\bar{x}_{n} \in S_{n}$ given in proposition 3.2 undergo border collision bifurcations. That is, curves for which the fixed points collide with the boundaries:

$$
\begin{aligned}
& A \rightarrow A_{n}^{\mathcal{L}}(d) \Longrightarrow \bar{x}_{n} \longrightarrow\left(\Sigma_{n}\right)^{-} \\
& A \rightarrow A_{n}^{\mathcal{R}}(d) \Longrightarrow \bar{x}_{n} \longrightarrow\left(\Sigma_{n-1}\right)^{+} .
\end{aligned}
$$

Proposition 3.3. For any $0<d<1$ there exists a sequence

$$
0<A_{0}(d)<A_{1}^{\mathcal{R}}(d)<A_{1}^{\mathcal{L}}(d)<A_{2}^{\mathcal{R}}(d)<A_{2}^{\mathcal{L}}(d)<\ldots,
$$

such that, for every $n>0$, the stroboscopic map $\mathfrak{s}$ (3.7) possesses a unique fixed point $\bar{x}_{n} \in S_{n}$ for $A \in\left(A_{n}^{\mathcal{R}}(d), A_{n}^{\mathcal{L}}(d)\right)$. This fixed point undergoes a border collision bifurcation at $A=A_{n}^{\mathcal{L}}(d)$ and $A=A_{n}^{\mathcal{R}}(d)$.

The values $A_{0}(d), A_{n}^{\mathcal{L}}(d)$ and $A_{n}^{\mathcal{R}}(d)$ define smooth curves fulfilling

$$
\begin{aligned}
& \lim _{d \rightarrow 0} A_{0}=\infty \\
& \lim _{d \rightarrow 0} A_{n}^{\mathcal{R}, \mathcal{L}}(d)=\infty
\end{aligned}
$$

Hence these curves go through the origin of the parameter space $(d, 1 / A)$. Moreover,

$$
\lim _{d \rightarrow 1} A_{n}^{\mathcal{R}}(d)=\lim _{d \rightarrow 1} A_{n}^{\mathcal{L}}(d), n \geq 1,
$$


and this limit becomes the value of $A$ for which

$$
\delta=\frac{T}{n}
$$

where $\delta$ is defined in eq. (3.4).

Proof. Let $\mathfrak{s}_{-}$and $\mathfrak{s}_{+}$be as in eqs. (3.11) and (3.12):

$$
\begin{aligned}
& \mathfrak{s}_{-}=\varphi(T(1-d) ; \theta ; 0) \\
& \mathfrak{s}_{+}=\varphi(T(1-d) ; 0 ; 0) .
\end{aligned}
$$

As given in lemma 3.3. if the stroboscopic map exhibits a discontinuity, $\Sigma_{n} \in$ $(0, \theta)$, then these values coincide with the lateral images of $\Sigma_{n}$ :

$$
\begin{aligned}
\mathfrak{s}\left(\Sigma_{n}^{-}\right) & =\mathfrak{s}_{-} \\
\mathfrak{s}\left(\Sigma_{n}^{+}\right) & =\mathfrak{s}_{+} .
\end{aligned}
$$

For $0<d<1$ and $n \geq 1$, let $A_{n}^{\mathcal{C}}(d)$ be as in the proof of prop. 3.2 i.e. the value of $A$ defined by the condition $\Sigma_{n}=0$. By lemma 3.2 and the implicit function theorem $A_{n}^{\mathcal{C}}(d)$ is a smooth function of $d$. We begin by discussing the bifurcation sequence occurring for some $0<d<1$ and $0<A<A_{1}^{\mathcal{C}}(d)$. The sequence of bifurcations that we now describe are illustrated in fig. 9 for a particular example.

First note that for $A$ sufficiently small, $[0, \theta)=S_{0} \cup S_{1}$ and $\Sigma_{1}>\mathfrak{s}_{-}=\mathfrak{s}\left(\Sigma_{1}^{-}\right)$. In this case the graph of $\mathfrak{s}$ is as shown in figure 6(c) (for $n=3$ ) and clearly the fixed point $\bar{x}_{0}$ contained in $S_{0}$ must exist. By lemma 3.2 , when $A$ is increased $\Sigma_{1}$ decreases with non-zero speed. Hence there exists a unique value of $A=A_{0}$ such that $\Sigma_{1}=\mathfrak{s}_{-}=\mathfrak{s}\left(\Sigma_{1}^{-}\right)$(see figure 6(d) for $\Sigma_{3}$ ). In other words, the fixed point $\bar{x}_{0}$ undergoes a border collision bifurcation as it collides with the boundary $\Sigma_{1}$ on its left. Moreover, by lemma 3.2 and the implicit function theorem, the equation $\Sigma_{1}=\mathfrak{s}_{-}$defines a smooth function $A_{0}(d)$.

For $A>A_{0}(d)$ the map $\mathfrak{s}$ is as shown in figure $4(\mathrm{~b})$ and the fixed point $\bar{x}_{0}$ no longer exists. As $A$ is further increased $\Sigma_{1}$ crosses $\mathfrak{s}_{+}$for some value $A=A_{1}^{\mathcal{R}}$. For this value, the fixed point $\bar{x}_{1}$ undergoes a border collision bifurcation as it collides with the boundary $\Sigma_{1}$ on its right (see figure 6(a) for $\bar{x}_{2}$ ). Similarly as above, the equation $\Sigma_{1}=\mathfrak{s}_{+}$defines a smooth function $A=A_{1}^{\mathcal{R}}(d)$ and for $A>A_{1}^{\mathcal{R}}(d)$ the fixed point $\bar{x}_{1}$ exists. Finally, for $A=A_{1}^{\mathcal{C}}(d)$ the map is continuous on the entire $(0, \theta)$ with fixed point $\bar{x}_{1}$.

We now repeat the same argument to show that the same bifurcation sequence occurs for $A_{n}^{\mathcal{C}}(d)<A \leq A_{n+1}^{\mathcal{C}}(d), n \geq 1$. Indeed, for $A>A_{n}^{\mathcal{C}}(d)$ but close to $A_{n}^{\mathcal{C}}(d)$ we have $\Sigma_{n}>\mathfrak{s}_{-}$. In this case the graph of $\mathfrak{s}$ is again as shown in figure 6(c) and clearly the fixed point $\bar{x}_{n-1}$ contained in $S_{n-1}$ must exist. By lemma 3.2, when $A$ is increased $\Sigma_{n}$ decreases with non-zero speed. Hence there exists a unique value of $A=A_{n-1}^{\mathcal{L}}$ such that $\Sigma_{n}=\mathfrak{s}_{-}=\tilde{\mathfrak{s}}\left(\Sigma_{n}^{-}\right)$(see figure $6(\mathrm{~d}$ ) for $n=3)$. For this value of $A$, the fixed point $\bar{x}_{n-1}$ undergoes a border collision as it collides with the boundary $\Sigma_{n}$ on its left. Moreover, by lemma 3.2 and 
the implicit function theorem, the equation $\Sigma_{n}=\mathfrak{s}_{-}=\mathfrak{s}\left(\Sigma_{n}^{-}\right)$defines a smooth function $A_{n}^{\mathcal{L}}(d)$.

For $A>A_{n-1}^{\mathcal{L}}(d)$ the map $\mathfrak{s}$ is as shown in figure $4(\mathrm{~b})$ and the fixed point $\bar{x}_{n-1}$ no longer exists. As $A$ is further increased, there exists some value $A=A_{n}^{\mathcal{R}}(d)$ for which $\Sigma_{n}$ crosses $\mathfrak{s}_{+}$, as in figure 6(a) For this value, the fixed point $\bar{x}_{n}$ undergoes a border collision bifurcation as it collides with the boundary $\Sigma_{n}$ on its right. Similarly as above the equation $\Sigma_{n}=\mathfrak{s}_{+}=\mathfrak{s}\left(\Sigma_{n}^{+}\right)$defines a smooth function $A=A_{n}^{\mathcal{R}}(d)$ and for $A>A_{n}^{\mathcal{R}}(d)$ the fixed point $\bar{x}_{n}$ exists. Finally, for $A=A_{n}^{\mathcal{C}}(d)$ the map is continuous on the entire $(0, \theta)$ with fixed point $\bar{x}_{n}$.

Finally, note that due to the fact that the lateral values of $\Sigma_{n}$ by $\mathfrak{s}\left(\mathfrak{s}_{-}\right.$and $\mathfrak{s}_{+}$) do not depend on $A$ and, by lemma $3.2, \Sigma_{n}$ monotonically decreases with $A$, the bifurcations described above are unique, as $\mathfrak{s}\left(\Sigma_{n}^{ \pm}\right)=\mathfrak{s}_{ \pm}$can occur only once.

As will be shown in next section, the periodic orbits that exist in the intervals of the form $\left(A_{n-1}^{\mathcal{L}}(d), A_{n}^{\mathcal{R}}(d)\right)$ are given by the period adding strucutre. $d \rightarrow 1$.

We now discuss the limiting values of the curves $A_{n}^{\mathcal{R}, \mathcal{L}}(d)$ when $d \rightarrow 0$ and

Let $A_{0}(d)$ be the curve where the fixed point $\bar{x}_{0} \in S_{0}$ undergoes a border collision. As discused above, this occurs when the fixed point $\bar{x}_{0}$ collides with the boundary:

$$
\begin{gathered}
\varphi\left(d T ; \bar{x}_{0} ; A_{0}\right)=\theta \\
\varphi(T-d T ; \theta ; 0)=\bar{x}_{0},
\end{gathered}
$$

where $\varphi(t ; x ; A)$ is the flow associated with system $\dot{x}=f(x)+A$. Due to the monotonicity of $f(x), \varphi(d T ; x ; A)$ is a monotonotonically increasing function of $A$ for $A \in\left[A_{0}, A_{1}^{\mathcal{R}}\right)$ for all $x \in[0, \theta)$. Hence, if $d \rightarrow 0$ then $A_{0} \rightarrow \infty$ in order to keep equation (3.16) satisfied. Therefore the curve defined by $A_{0}(d)$ goes through the origin of the parameter space $d \times 1 / A$. The sequence given in eq. 3.15 implies that all the other bifurcation curves given by $A_{n}^{\mathcal{R}, \mathcal{L}}(d)$ also go through this point.

We now focus on $d \rightarrow 1$. Let $A \in\left(A_{n}^{\mathcal{R}}(d), A_{n}^{\mathcal{L}}(d)\right)$ and hence there exists a fixed point of $\mathfrak{s}, \bar{x}_{n} \in S_{n}$. In order to see (3.3) we will show that, if there exists such a fixed point, then necessary $\mathfrak{s}(x) \rightarrow x$ when $d \rightarrow 1$; that is, if a fixed point exists, then the stroboscopic map $\mathfrak{s}$ tends to be the identity when $d \rightarrow 1$. If that's the case, then, both bifurcations tend to occur at the same time and hence

$$
A_{n}^{\mathcal{R}}(d)-A_{n}^{\mathcal{L}}(d) \rightarrow 0,
$$

and both limits exists, which proves eq. (3.3).

To see that $\mathfrak{s}$ tends to be the identity when $d \rightarrow 1$ if $\bar{x}_{n} \in S_{n}$ we first recall that, for $d=1$ the system (2.1)-(2.3) becomes the autonomous system $\dot{x}=f(x)+A$ plus the reset condition 2.3). As $A \in\left(A_{n}^{\mathcal{R}}(d), A_{n}^{\mathcal{L}}(d)\right)$ is large enough to make the system exhibit spikes, if $d=1$ there exists only a periodic orbit with period $\delta=\delta(A)$ defined in (3.4). Moreover, all points in $[0, \theta)$ belong to this periodic orbit because of the reset. As a consequence, all initial conditions in $[0, \theta)$ are 
fixed points of the time- $\delta$ return map and this map is hence the identity.

We now write the conditions for the existence of the fixed point $\bar{x}_{n}$ in terms of the flow:

$$
\begin{aligned}
\varphi\left(t_{n} ; \bar{x}_{n} ; A\right) & =\theta \\
\varphi\left(d T-t_{n}-(n-1) \delta ; 0 ; A\right) & =x^{\prime} \\
\varphi\left(T-d T ; x^{\prime} ; 0\right) & =\bar{x}_{n} .
\end{aligned}
$$

Clearly, when $d \rightarrow 1, x^{\prime} \rightarrow \bar{x}_{n}$ and $A$ tends to be such that $T$ is a multiple of $\delta(A)$; indeed

$$
n \delta(A) T \rightarrow T .
$$

As a consequence, for this limiting value of $A$, the time- $T$ return map, $\mathfrak{s}$, also tends to be the identity in $S_{n}$.

\subsection{Period adding structures}

In this section we study the invariant objects (periodic orbits) located in the regions in the parameter space $d \times 1 / A$ nested in between fixed points of the stroboscopic map (gray regions in fig. $1(\mathrm{a})$.

As announced in \$2, these are organized by period adding structures. Using the background on this topic provided by theorem 2.1, this is a direct consequence of the following proposition, which states that the stroboscopic map (3.7) can be written as the normal form given in eq. 2.7) for parameter values between two consecutive regions where fixed points exist. This will be a consequence of the fact that all fixed points simultaneously undergo border collision bifurcations at the origin of the parameter space $d \times 1 / A$.

Proposition 3.4. Let

$$
\begin{array}{rlc}
\mu:[0,1] & \longrightarrow & \mathbb{R}^{2} \\
\lambda & \longmapsto & (d(\lambda), B(\lambda))
\end{array}
$$

be a $C^{\infty}$ parametrization of a curve in the parameter space $d \times 1 / A$ such that $\mu(0)=\left(d_{0}, B_{0}\right)$ and $\mu(1)=\left(d_{1}, B_{1}\right)$ fulfilling

$$
\begin{aligned}
d_{i} & \in(0,1) \\
B_{0} & =\frac{1}{A_{n}^{\mathcal{L}}\left(d_{0}\right)} \\
B_{1} & =\frac{1}{A_{n+1}^{\mathcal{R}}\left(d_{1}\right)},
\end{aligned}
$$

for some $n>0$, and

$$
B^{\prime}(\lambda)<0, \lambda \in(0,1) .
$$

Let $\mathfrak{s}_{\lambda}$ be the map obtained by applying the reparametrization $\mu$ to $\mathfrak{s}$. Then, the bifurcation diagram associated with the map $\mathfrak{s}_{\lambda}$ for $\lambda \in[0,1]$ follows a period adding as described for the map 2.7. 
Proof. We show that the map $\mathfrak{s}$ can be written in the form of map (2.7) fulfilling the conditions of theorem 2.1

After performing the reparametrization $\lambda \longmapsto(d, A)$ given by the curve (3.17), we have that $\Sigma_{n}(\lambda) \in[0, \theta) \forall \lambda \in[0,1]$. Hence, the change of variables

$$
\tilde{x} \longmapsto x-\Sigma_{n}(\lambda),
$$

is well defined and makes the map $\tilde{\mathfrak{s}}_{\lambda}(\tilde{x}):=\mathfrak{s}\left(\tilde{x}+\Sigma_{n}(\lambda)\right)-\Sigma_{n}(\lambda)$ undergo a discontinuity at $\tilde{x}=0$, independently of $\lambda$. The stroboscopic map $\tilde{\mathfrak{s}}_{\lambda}$ is continuous, increasing and contracting in $\left[0, \theta-\Sigma_{n}(\lambda)\right)$ and $\left[-\Sigma_{n}(\lambda), 0\right)$ for $\lambda \in[0,1]$. Hence, the map $\tilde{\mathfrak{s}}_{\lambda}(\tilde{x})$ is of type $(2.7)$ and satisfies 2.9$]$ in its domain, which is enough as argued in remark 2.1.

As argued in the proof of proposition 3.3 the map $\tilde{\mathfrak{s}}_{\lambda}(\tilde{x})$ undergoes a negative gap at $\tilde{x}=0$ for $\lambda \in[0,1]$ :

$$
\tilde{\mathfrak{s}}_{\lambda}\left(0^{-}\right)>\tilde{\mathfrak{s}}_{\lambda}\left(0^{+}\right) \text {. }
$$

For $\lambda=0$ and $\lambda=1$, the fixed points $\bar{x}_{n-1}-\Sigma_{n}(0)$ and $\bar{x}_{n}-\Sigma_{n}(1)$ of the map $\tilde{\mathfrak{s}}_{\lambda}(\tilde{x})$ undergo border collision bifurcations. This implies

$$
\begin{aligned}
& \tilde{\mathfrak{s}}_{0}\left(0^{-}\right)=0, \\
& \tilde{\mathfrak{s}}_{1}\left(0^{+}\right)=0 .
\end{aligned}
$$

Hence, condition C.3 of theorem 2.1 is satisfied. Recalling the negative gap (3.18), the map $\tilde{\mathfrak{s}}_{\lambda}(\tilde{x})$ fulfills

$$
\begin{aligned}
& \tilde{\mathfrak{s}}_{\lambda}\left(0^{+}\right)<0, \forall \lambda \in(0,1), \\
& \tilde{\mathfrak{s}}_{\lambda}\left(0^{-}\right)>0, \forall \lambda \in(0,1),
\end{aligned}
$$

and hence C.1 in theorem 2.1 is satisfied too.

From the fact that $B^{\prime}(\lambda)<0$, the reparametrization is such that the parameter $A$ monotonically decreases with $\lambda$. From lemma 3.2 we get that $\Sigma_{n}(\lambda)$ also decreases monotonically with $\lambda$ and hence C.2 in theorem 2.1 is also satisfied.

Finally, we can apply theorem 2.1 for the normal form map 2.7 to obtain that the bifurcation scenario exhibited by the map $\tilde{\mathfrak{s}}_{\lambda}$ (an thus by the original map $\mathfrak{s}_{\lambda}$ ) when varying $\lambda$ is given by the period adding structure.

By combining the previous result and the bifurcation scenario described for the normal form map (2.7) we get that the regions in parameter space between curves of the form $\left(d, 1 / A_{n}^{\mathcal{L}}(d)\right)$ and $\left(d, 1 / A_{n+1}^{\mathcal{R}}(d)\right)$ are covered by an infinite number of periodic orbits with arbitrarily high period following the period adding structure described in $\$ 2$. In addition, by combining it with lemma 3.3 we get the full description of the bifurcation scenario for system (2.1)-2.3 in the parameter space $(d, 1 / A)$, as described in $\$ 2$. 


\subsection{Symbolic sequences, firing number and firing rate}

We now introduce the symbolic dynamics described in $\$ 2$ Let $\left(x_{1}, \ldots, x_{n}\right)$ be a $n$-periodic orbit located in the region in parameter space between the curves $\left(d, 1 / A_{n}^{\mathcal{L}}\right)$ and $\left(d, 1 / A_{n+1}^{\mathcal{R}}\right)$. We then assign to this orbit the symbolic sequence given by the encoding

$$
\begin{array}{ll}
x_{i} \rightarrow \mathcal{L} & \text { if } x_{i}<\Sigma_{n} \\
x_{i} \rightarrow \mathcal{R} & \text { if } x_{i} \geq \Sigma_{n} .
\end{array}
$$

Note that $x_{i}<0$ and $x_{i} \geq 0$ correspond to $x_{i} \in S_{n}$ and $x_{i} \in S_{n+1}$, respectively. Hence, recalling the definition of the sets $S_{i}$ provided in eq. (3.8), when introducing the encoding (2.8), the symbols $\mathcal{L}$ and $\mathcal{R}$ have to be interpreted as the system spiking $n$ and $n+1$ times for one iteration of the stroboscopic map, respectively. This determines the so-called firing number, introduced in KHR81. as follows.

Definition 3.1. Let $s$ be the total number of spikes performed by a p-periodic orbit of the stroboscopic map $\mathfrak{s}(x)$ when iterated $p$ times, $n, p \in \mathbb{N}$; then we define the firing-number as

$$
\eta=\frac{s}{p} .
$$

Provided that these periodic orbits are attractive, this number becomes the asymptotic average number of spikes per iteration of the stroboscopic map.

Note that this quantity can be computed from the symbolic sequence. Let $\sigma$ be a $p$-periodic orbit for parameter values located between the curves $1 / A=$ $1 / A_{n}^{\mathcal{L}}(d)$ and $1 / A=1 / A_{n+1}^{\mathcal{R}}$ whose symbolic sequence contains $m \mathcal{R}^{\prime} s$ and $k \mathcal{L}^{\prime} s$ $(k=p-m)$. Then, the firing number becomes

$$
\eta=\frac{n k+(n+1) m}{p}=n+\frac{m}{p} .
$$

As explained in 92 , the rotation numbers associated with the periodic orbits in the period adding structure can be computed dividing the number of $\mathcal{R}^{\prime} s$ symbols in its symbolic sequence by its total period,

$$
\rho=\frac{m}{p} \text {. }
$$

Hence, the firing number can be related with the rotation number as

$$
\eta=n+\rho,
$$

where $n$ is such that the periodic orbit steps on each side of the boundary $\Sigma_{n}$. As explained also in $\$ 2$, the rotation number is organized by the Farey tree structure shown in fig. 3(a) when parameters are varied from the bifurcation curve $1 / A=1 / A_{n}^{\mathcal{L}}(d)$ towards $1 / A=1 / A_{n+1}^{\mathcal{R}}$ along curves as the one given in proposition 3.4. Hence, it follows a devil's staircase from 0 to 1 . As a consequence, the firing number follows a devil's staircase from $n$ to $n+1$ when parameters $d$ and $A$ are varied as mentioned. 
Taking into account that the stroboscopic map consists of flowing system (2.1)2.3 for a time $T$, the firing number allows one to compute the firing rate associated with the corresponding periodic orbit as

$$
r=\frac{\eta}{T} .
$$

This becomes the asymptotic number of spikes per unit time.

Note that, provided that these periodic orbits are attracting, $r$ becomes the asymptotic firing rate defined in eq. 2.6),

$$
r=\lim _{\tau \rightarrow \infty} \frac{\left.\# \text { (spikes performed by } \phi\left(t ; x_{0}\right) \text { for } t \in[0, \tau]\right)}{\tau},
$$

which does not depend on $x_{0} \in[0, \theta)$.

Using relation given in eq. $(3.20)$, the firing rate follows also a devil's staircase from $n / T$ and $(n+1) / T$ when the parameter values are varied through curves as the one in prop. 3.4 between the bifurcation curves $1 / A=1 / A_{n}^{\mathcal{L}}(d)$ and $1 / A=1 / A_{n+1}^{\mathcal{R}}(d)$, respectively.

\section{Examples}

In this section we illustrate the results presented so far in two different examples. We consider a system of the type

$$
\dot{x}=f(x)+I(t),
$$

with $I(t)$ a $T$-periodic input as defined in eq $(2.2)$ and three different types of functions for $f(x)$ satisfying conditions H.1-H.2. The first example consists of a linear system, leading to the so-called linear integrate-and-fire system (LIF):

$$
f_{1}(x)=a x+b .
$$

Taking the threshold $\theta=1$, conditions H.1-H.2 are satisfied if $a<0$ and $-b / a \in$ $(0,1)$. In figure 7 we show the existence of periodic orbits in the parameter space $d \times 1 / A$, which is as predicted. In fig. $8(\mathrm{a})$ we show the periods of the periodic orbits found along the line drawn in figure 7, although the bifurcation scenario is topologically the same for any other curve transversally crossing the colored regions. As one can see in fig. 8(a), along such a curve there exist regions where one finds only $T$-periodic orbits (fixed points of the stroboscopic map, period 1 ). These are the black regions in figure 7, for example containing the points as $B, C, D$ and $E$. The periodic orbits for the parameter values for $B$ and $C$ are shown in figs. $9(\mathrm{a})$ and $9(\mathrm{~d})$ respectively. Note that their associated firing number defined in eq. (3.20) (average number of spikes per iteration of the stroboscopic map) is 0 and 1 , respectively.

As given by propositions 3.2 and 3.3 , there exist an infinite number of regions, accumulating at the horizontal axis $1 / A=0$, for which one finds $T$-periodic orbits with arbitrarily large integer firing number. This can be seen in figure $8(\mathrm{~b})$ 


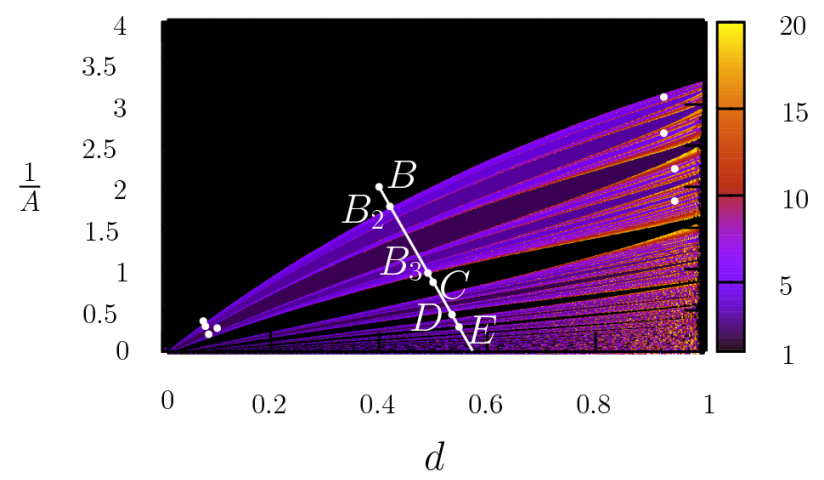

Figure 7: Bifurcation diagram for the map $\mathfrak{s}$ for $T=1.9$ associated with system 2.1)-2.3 for the linear system (4.1). The colors refer to the periods of the periodic orbits found by simulating the system. Periodic orbits are shown only up to period 20 for clarity reasons. The $T$-periodic orbits (fixed points of $\mathfrak{s}$ ) for parameter values corresponding to points $B$ and $C$, as well as their bifurcations $\left(B_{2}\right.$ and $\left.B_{3}\right)$, are shown in fig. 9. The non-labeled eight points correspond to $5 T$-periodic orbits with four different symbolic sequences. The ones for the four points with small values of $d$ are shown in fig. 10, and the ones with large values of $d$ in fig. 11 .

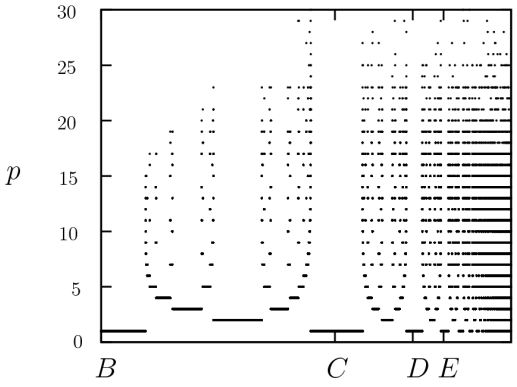

(a)

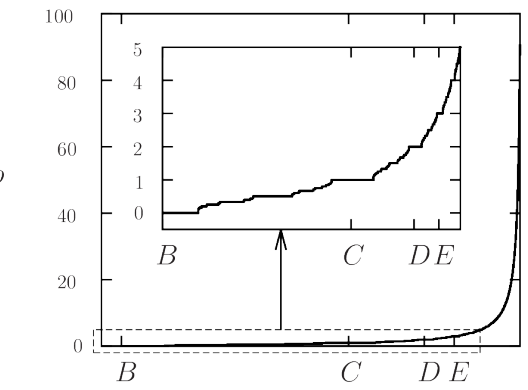

(b)

Figure 8: (a) periods of the periodic orbits found along a line as the one shown in fig. 7. (b) firing number associated with such periodic orbits. 
for the points $D$ and $E$ and successive regions containing $T$-periodic orbits (period one figure 8(a)).

The two $T$-periodic orbits found in $B$ and $C$ undergo border collision bifurcations at the points $B_{2}$ and $B_{3}$ labeled in fig. 7. The periodic orbits at the moment of the bifurcation are shown in figures 9(b) and 9(c), respectively. This occurs similarly for all other $T$-periodic orbits with higher firing number (see proposition 3.3 for more details). In between points $B$ and $C, C$ and $D, D$ and $E$ etc., a period adding bifurcation occurs; that is, there exist an infinite number of periodic orbits whose periods, symbolic sequences and rotation numbers are organized by the Farey tree structure shown in fig. 3(a) After applying the symbolic encoding (2.8), the symbolic sequences and periods are obtained by successive concatenation and addition as shown in fig. 3(a) and explained in section 2. In figures 10 and 11 we show, for different parameter values, the four periodic orbits with period 5 located in the adding structure between points $B$ and $C$, which correspond to the symbolic sequences $\mathcal{L}^{4} \mathcal{R}, \mathcal{L}^{2} \mathcal{R} \mathcal{L} \mathcal{R}, \mathcal{L} \mathcal{R} \mathcal{L}{ }^{2}$ and $\mathcal{L R}^{4}$ (see the Farey tree in fig. 3(a)). The parameter values used are given by the points marked in fig. 7, for large and small $d$. Note how the $\mathcal{L}$ symbol corresponds to an iteration of the stroboscopic map without exhibiting any spike, whereas for $\mathcal{R}$ one spike occurs.

The firing number associated with the periodic orbits is related with their rotation number through equation 3.20), and it is shown in figure 8(b) for the periodic orbits found the line labeled in fig. 7. As one can see there and as it comes from proposition 3.4 (see $\S 3.4$ ), the firing number is a strictly increasing and unbounded devil's staircase as a function of the parameters.

Finally, we show that the same results apply for system (2.1)-(2.3) with $f(x)$ not necessary linear as long as conditions H.1-H.2 are satisfied. To illustrate this we choose two different functions for $f$ :

$$
\begin{aligned}
& f_{2}(x)=a_{2}\left(x-b_{2}\right)^{5}-c_{2} x \\
& f_{3}(x)=-\arctan \left(a_{3}\left(x-b_{3}\right)\right) .
\end{aligned}
$$

With $\theta=1$, we choose parameter values in order to make the functions $f_{2}$ and $f_{3}$ satisfy condition H.1-H.2:

$$
\begin{array}{ll}
a_{2}=-10 & a_{3}=100 \\
b_{2}=0.7 & b_{3}=0.1 \\
c_{2}=0.01 &
\end{array}
$$

These two functions are shown for these parameter values in fig. 12 There one can see that the first case corresponds to a weak equilibrium point whereas the second one to a robust one. This leads to slow and fast subthreshold dynamics, respectively.

The bifurcation structures in the parameter space $d \times 1 / A$ are shown in fig. 13 , which are as predicted. The bifurcation scenarios along curves transversally 


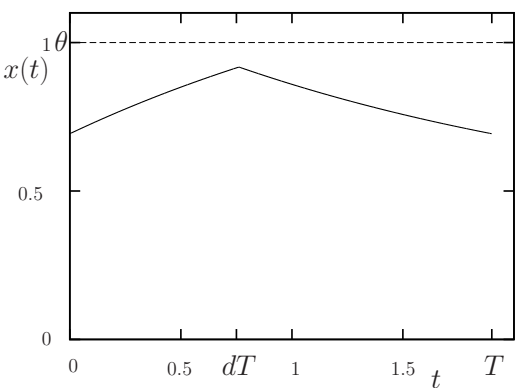

(a)

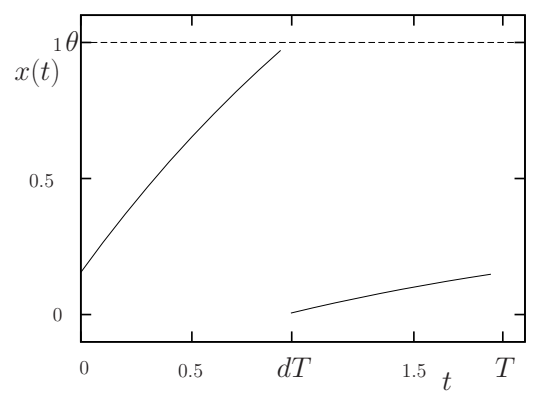

(c)

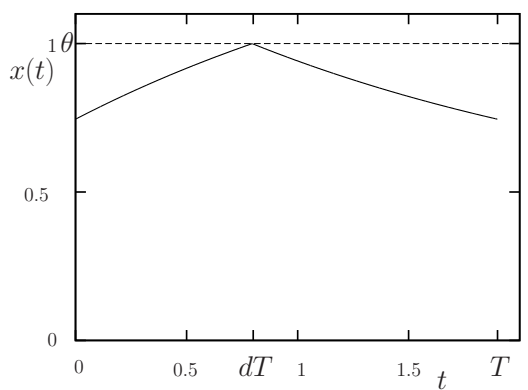

(b)

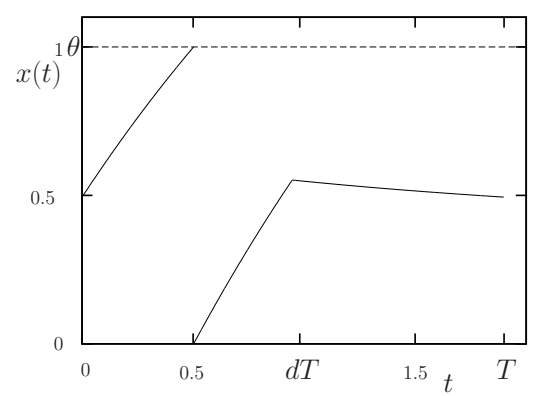

(d)

Figure 9: Periodic orbits for the labeled points in fig. 7) (a) periodic orbit with no spikes (fixed point $\bar{x}_{0}$ ), (b) left bifurcation of the fixed point, $\bar{x}_{0}=\Sigma_{1}$, (c) right bifurcation of the fixed point $\bar{x}_{1}=\Sigma_{1}$, (d) periodic orbit spiking once. 


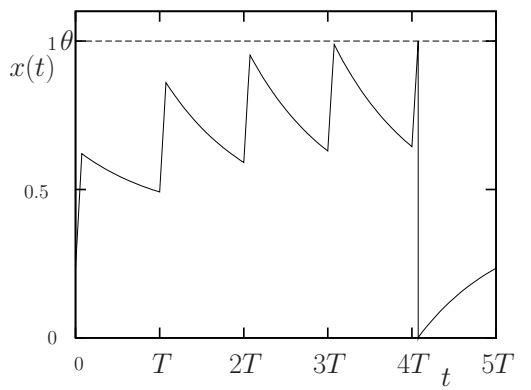

(a)

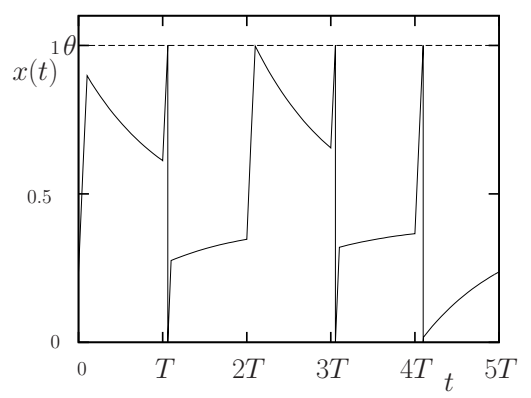

(c)

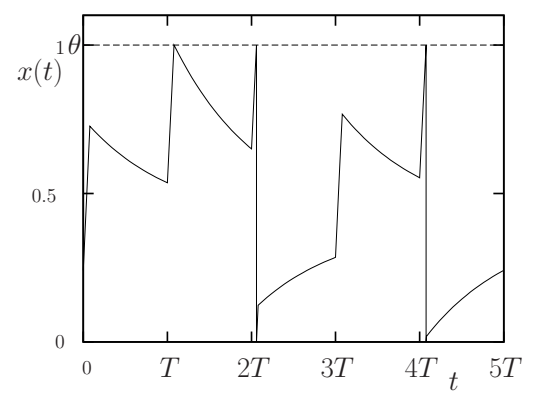

(b)

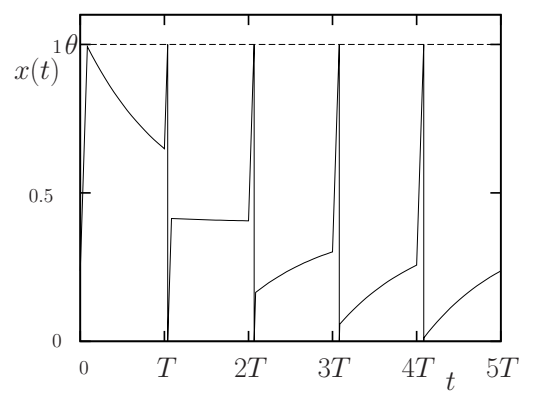

(d)

Figure 10: Period-5 periodic orbits with different symbolic sequences and firing number: $\mathcal{L}^{4} \mathcal{R} \eta=1 / 5(\mathrm{a}), \mathcal{L}^{2} \mathcal{R} \mathcal{L} \mathcal{R} \eta=2 / 5$ (b), $\mathcal{L} \mathcal{R} \mathcal{L}^{2} \eta=3 / 5$ (c) and $\mathcal{L R}^{4}$ $\eta=4 / 5$ (d). Parameter values for which these periodic orbits exist are marked with points in figure 7 , and correspond to small values of the duty cycle $d$. 


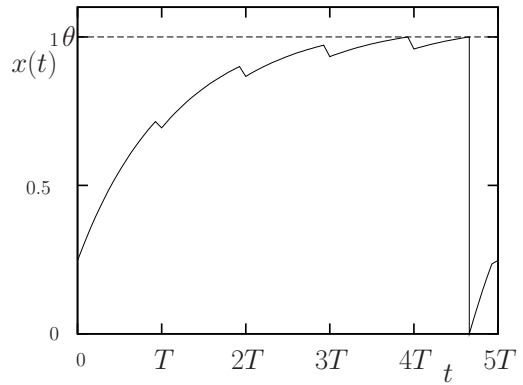

(a)

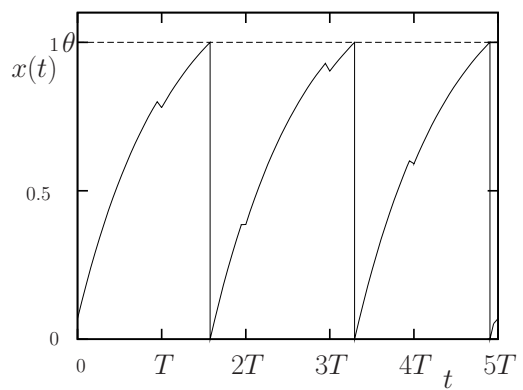

(c)

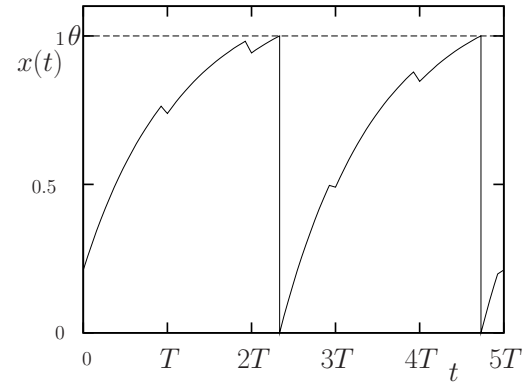

(b)

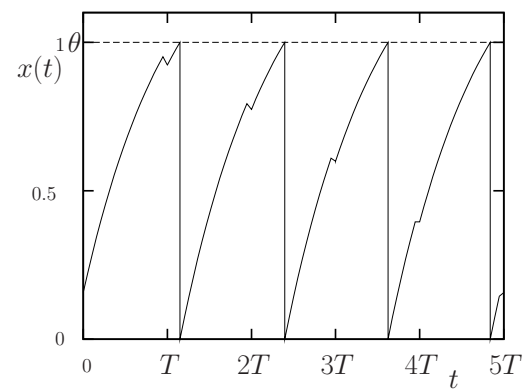

(d)

Figure 11: Period 5 periodic orbits with different symbolic sequences and firing number: $\mathcal{L}^{4} \mathcal{R} \eta=1 / 5$ (a), $\mathcal{L}^{2} \mathcal{R} \mathcal{L} \mathcal{R} \eta=2 / 5$ (b), $\mathcal{L} \mathcal{R} \mathcal{L R}^{2} \eta=3 / 5$ (c) and $\mathcal{L R}^{4}$ $\eta=4 / 5$ (d). Parameter values for which these periodic orbits exist are marked with points in figure 7, and correspond to large values of the duty cycle $d$.

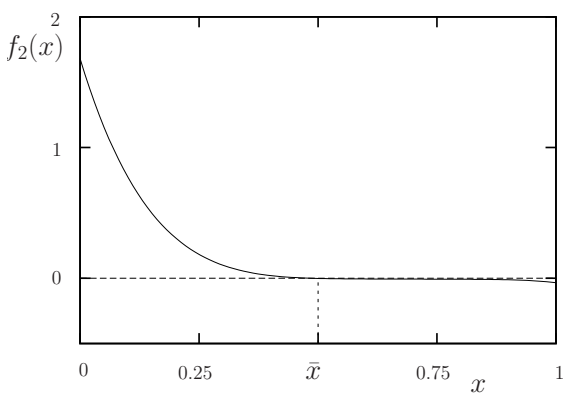

(a)

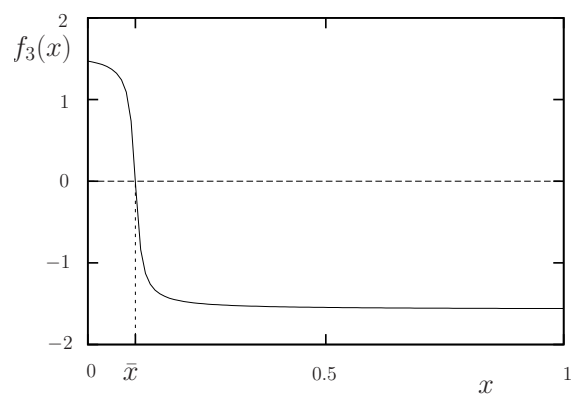

(b)

Figure 12: Functions $f_{2}$ and $f_{3}$ given in eq. $(4.2) . \bar{x}$ is the attracting equilibrium point. 


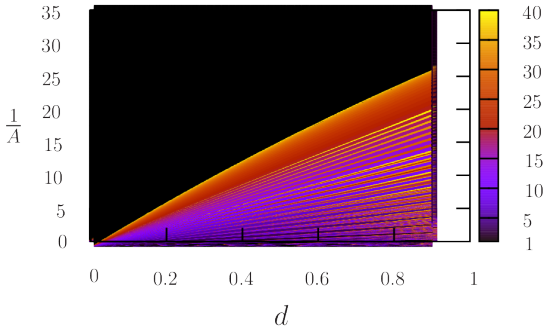

(a)

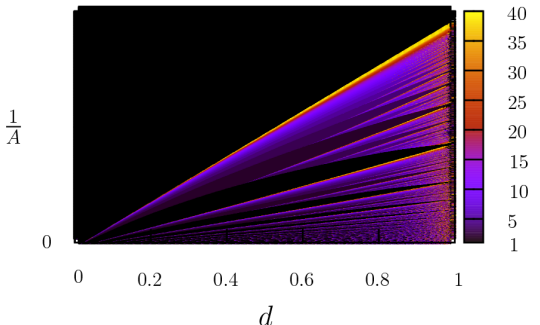

(b)

Figure 13: Bifurcation scenarios for the second and third examples given in eq. (4.2). (a) and (b) correspond to $f_{2}$ and $f_{3}$, respectively. The values of the period $T$ have been $T=1$ for (a) and $T=0.5$ for (b).

crossing the bifurcation curves are equivalent to the ones for the first example, as predicted by our results, and we do not show further details.

The bifurcation scenario in the $d \times 1 / A$ plane for the weak equilibrium point is mainly covered by periodic orbits (bursting spiking), whereas in the second case, the spiking dynamics is mainly given by fixed points of the stroboscopic (tonic spiking). This is because the subthreshold dynamics for $f_{2}$ is much slower than for $f_{3}$. This can be seen in fig. 14, where we show for both systems a 5periodic orbit with symbolic sequence $\mathcal{L} \mathcal{R} \mathcal{L R}^{2}$. Note that the value of $T$ for $f_{3}$ in fig. 13(b) had to be reduced with respect to fig. 13(a) in order to observe bursting spiking (periodic orbits), because, due to the fast subthreshold dynamics, for the same value of $T$ as for $f_{2}$ one basically observes tonic spiking (fixed points). When reducing the period, the fast convergence of the subtreshold dynamics for $f_{3}$ is compensated and hence the regions in parameter space locating fixed points become broauder and easier observable. We refer to [GKC13, for a complete study of the behaviour of the bifurcation curves under frequency variation of the input.

\section{Discussion}

Hybrid systems with resets are a simplified version of excitable systems and are widely used in modeling of biological systems, e.g. in the context of neuronal activity and secretion of hormones. Typically, as such systems undergo discontinuities (spikes), they are studied by means of firing maps, or impact maps, i.e. Poincaré maps defined using the threshold as a Poincaré section. In this work we have studied generic periodically forced hybrid systems under the assumption of monotonicity and contracting dynamics. The main innovation of this paper was to use the stroboscopic (time $T$ return map) rather than the return 


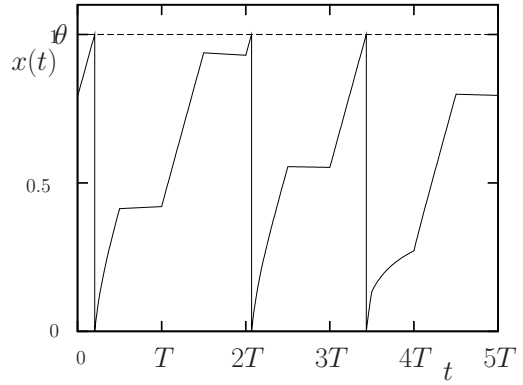

(a)

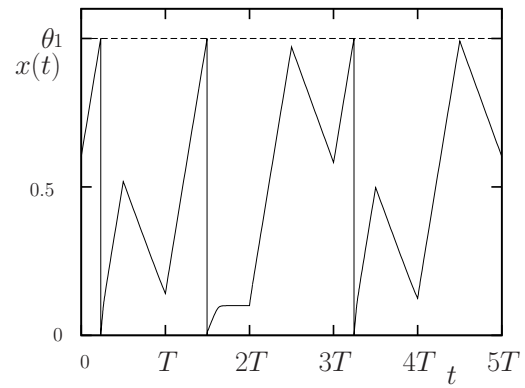

(b)

Figure 14: Period-5 periodic orbits with symbolic sequence $\mathcal{L} \mathcal{R} \mathcal{L R}^{2}$ and firing number $\eta=3 / 5$ for the examples given in eq. 4.2). (a) corresponds to $f_{2}$ and (b) to $f_{3}$. Parameter values are $1 / A=0.79$ and $1 / A=0.2$ for (a) and (b), respectively, and $d=0.5$ in both cases.

map. We have obtained a complete description of the bifurcation structure and the dynamics, showing the existence of a globally attracting periodic orbit for every parameter value as well as giving a description of the transitions between different regions of stable periodicity. We have also introduced the rotation number and shown that its typical dependence on parameters is a devil's staircase. Finally, we defined firing rate in the context of these systems and related it to the rotation number.

It is important and interesting to compare the method developed in this paper with the approach based on the firing map. The main reason why the firing map has been the tool of choice is that in certain contexts it becomes a regular map; however, the analysis relies on explicit computation of the firing times and hence this method may not be suitable to provide general results that can be systematically applied. Firing maps become even more difficult to use in the presence of time dependent periodic forcings, as one needs to check for congruency between spiking times and the period of the forcing.

The property of smoothness, which is the main advantage of the firing map, holds automatically for systems in one dimension as the threshold is a real number which is identified with a different real number by means of the reset. However, in higher dimensions, (when considering dynamical adaption currents or dynamical thresholds) the threshold becomes a codimension-one manifold and one can find much richer dynamics. In fact, the firing map may exhibit discontinuities near those points where the flow exhibits tangencies with the threshold. As shown in [CTW12, $\mathrm{JMB}^{+} 13$, for two-dimensional linear systems that can be solved explicitly, the firing map may exhibit opposite slopes on both sides of such discontinuities. In this case, one can apply the results for piecewisesmooth maps given in AGS11] to obtain a full description of the bursting spiking 
dynamics (periodic orbits of the map) and its symbolic dynamics.

The advantage of using the stroboscopic map when considering periodically forced systems with resets is that, after proper reparametrizations, it can be classified as a discontinuous map with positive slopes near the discontinuity. Such maps have been extensively studied and are well understood [CGT84, GGT84, GIT84, GPTT86, Gam87, PTT87, TS86, GGT88, GT88, LPZ89, AS06. This allows us to provide general results that can be systematically applied to systems satisfying generic conditions. Moreover, the whole analysis of the period adding bifurcation structures comes from understanding only the bifurcations undergone by fixed points, and is independent of the number of spikes exhibited by the periodic orbits corresponding to these fixed points for the time continuous system. Finally, the presented framework is well suited for generalizations of the considered systems. For example, a similar analysis can be done for general periodic forcings, systems with expansive subthreshold dynamics or even systems in higher dimensions, as could be the case of considering a dynamical threshold.

\section{Acknowledgements}

We would like thank Jean-Marc Gambaudo for his helpful comments on discontinuous maps.

\section{References}

[AGS11] V. Avrutin, A. Granados, and M. Schanz. Sufficient conditions for a period increment big bang bifurcation in one-dimensional maps. Nonlinearity, 24(9):2575, 2011.

[AS06] V. Avrutin and M. Schanz. On multi-parametric bifurcations in a scalar piecewise-linear map. Nonlinearity, 19:531-552, 2006.

[BG05] R. Brette and W. Gerstner. Adaptive exponential integrate-and-fire model as an effective description of neuronal activity. J. Neurophysiol, 94:3637-3642, 2005.

[BM61] N.N. Bogoliubov and Y.A. Mitropolski. Asymptotic methods in the theory of non-linear oscillations. Gordon and Breach, 1961.

[CB99] S. Coombes and P.C. Bressloff. Mode locking and arnold tongues in integrate-and-fire neural oscillators. Phys. Rev E., 60:2086-2096, 1999.

[CGT84] P. C. Coullet, J. M. Gambaudo, and C. Tresser. Une nouvelle bifurcation de codimension 2: le colage de cycles. C. R. Acad. Sc. Paris, série I, 299:253-256, 1984. 
[COS01] S. Coombes, M. Owen, and G.D. Smith. Mode locking in a periodically forced integrate-and-fire-or-burst neuron model. Phys. Rev E., 64:041914, 2001.

[CPF08] J.R. Clay, D. Paydarfar, and D.B. Forger. A simple modification of the hodgkin and huxley equations explains type 3 excitability in squid giant axons. J. R. Soc. Interface, 5:1421-1428, 2008.

[CTW12] S. Coombes, R. Thul, and K.C.A Wedgwood. Nonsmooth dynamics in spiking neuron models. Physica D, 241:2042-2057, 2012.

[dBBCK08] M. di Bernardo, C. J. Budd, A. R. Champneys, and P. Kowalczyk. Piecewise-smooth Dynamical Systems: Theory and Applications, volume 163 of Applied Mathematical Sciences. Springer, 2008.

[DKTG12] M. Diopoppa, M. Krupa, A. Torcini, and B.S. Gutkin. Splay states in finite pulse-coupled networks of excitable neurons. SIAM J. Appl. Dyn. Syst. (SIADS), 11:3:864-894, 2012.

[Fit61] R. FitzHugh. Impulses and physiological states in models of nerve membrane. Biophys. J., 1:445-466, 1961.

[Gam87] J.M. Gambaudo. Ordre, dsordre, et frontiére des systmes MorseSmale. PhD thesis, Universit de Nice, 1987.

[GGT84] J.M. Gambaudo, P. Glendinning, and C. Tresser. Collage de cycles et suites de Farey. C. R. Acad. Sc. Paris, série I, 299:711-714, 1984.

[GGT88] J.M. Gambaudo, P. Glendinning, and T. Tresser. The gluing bifurcation: symbolic dynamics of the closed curves. Nonlinearity, 1:203-14, 1988.

[GH83] J. Guckenheimer and P. J. Holmes. Nonlinear Oscillations, Dynamical Systems and Bifurcations of Vector Fields. Appl. Math. Sci. Springer, 4th edition, 1983.

[GIT84] J.M. Gambaudo, O.Lanford III, and C. Tresser. Dynamique symbolique des rotations. C. R. Acad. Sc. Paris, série I, 299:823-826, 1984 .

[GKC13] A. Granados, M. Krupa, and F. Clément. Frequency decoding subject to dose conservation: firing rate response of periodically driven spiking models. Preprint available at https://who.rocq. inria.fr/Albert.Granados/, 2013.

[GPTT86] J.M. Gambaudo, I. Procaccia, S. Thomae, and C. Tresser. New Universal Scenarios for the Onset of Chaos in Lorenz-Type Flows. Phys. Rev. Lett, 57:925-928, 1986.

[GT88] J. Gambaudo and C. Tresser. On the dynamics of quasicontractions. BOL. SOC. BRAS. MAT., 19:61-114, 1988. 
[HH52] A. L. Hodgkin and A. F. Huxley. A Quantitative Description of Membrane Current and its Application to Conduction and Excitation in Nerve. Journal of Physiology, 117:500-544, 1952.

[Izh03] E.M. Izhikevich. Simple model of spiking neurons. IEEE Trans. Neur. Networks, 6:1569-1572, 2003.

[Izh07] E. M. Izhikevich. Dynamical Systems in Neuroscience: The Geometry of Excitability and Bursting. 2007.

$\left[\mathrm{JMB}^{+} 13\right]$ N.D Jimenez, S. Mihalas, R. Brown, E. Niebur, and J. Rubin. Locally contractive dynamics in generalized integrate-and-fire neurons. SIAM J. Appl. Dyn. Syst. (SIADS), 12:1474-1514, 2013.

[KHR81] J.P. Keener, F.C Hoppensteadt, and J. Rinzel. Integrate-and-fire models of nerve membrane response to oscillatory input. SIAM J. Appl. Dyn. Syst. (SIADS), 41:503-517, 1981.

[LPZ89] D. V. Lyubimov, A. S. Pikovsky, and M. A. Zaks. Universal Scenarios of Transitions to Chaos via Homoclinic Bifurcations, volume 8 of Math. Phys. Rev. Harwood Academic, London, 1989. Russian version 1986 as a Preprint (192) of Russian Academy of Science, Institute of mechanics of solid matter, Sverdlovsk.

[MHR12] X. Meng, G. Huguet, and J. Rinzel. Type III excitability, slope sensitivity and coincidence detection. Disc. Cont. Dyn. Syst., 32:2720$2757,2012$.

[ML81] L. Morris and H. Lecar. Voltage oscillations in the barnacle giant muscle fiber. Journ. Biophys., 35:193-213, 1981.

[NAY62] J. Nagumo, S. Arimoto, and S. Yoshizawa. An active pulse transmission line simulating nerve axon. Proc. IRE, 50:2061-2070, 1962.

[PTT87] I. Procaccia, S. Thomae, and C. Tresser. First-return maps as a unified renormalization scheme for dynamical systems. Phys. Rev. A, 35:1884-1900, 1987.

[TB09] J. Touboul and R. Brette. Spiking dynamics of bidimensional integrate-and-fire neurons. SIAM J. Appl. Dyn. Syst. (SIADS), 4:1462-1506, 2009.

[TS86] D. V. Turaev and L. P. Shil'nikov. On bifurcations of a homoclinic "Figure of Eight" for a saddle with a negative saddle value. Soviet Math. Dokl., 34:397-401, 1987 (Russian version 1986). 